\title{
Dynamic Multi-Priority, Multi-Class Patient Scheduling with Stochastic Service Times
}

\author{
Antoine Sauré $^{\mathrm{a}, *}$, Mehmet A. Begen ${ }^{\mathrm{b}}$, Jonathan Patrick ${ }^{\mathrm{a}}$ \\ ${ }^{a}$ Telfer School of Management, University of Ottawa, 55 Laurier Avenue East, Ottawa, ON, Canada K1N 6N5 \\ ${ }^{b}$ Ivey School of Business, Western University, 1255 Western Road, London, ON, Canada N6G ON1
}

\begin{abstract}
Efficient patient scheduling has significant operational, clinical and economical benefits on health care systems by not only increasing the timely access of patients to care but also reducing costs. However, patient scheduling is complex due to its stochastic nature, the existence of multiple stages of care, and the multiple interdependencies between these stages. Patient appointment (allocation) scheduling refers to the assignment of specific appointment start times to a set of patients scheduled for a particular day while advance patient scheduling refers to the assignment of future appointment days to patients. These two problems have generally been addressed separately despite each being highly dependent on the form of the other. This paper develops a framework that seeks to bridge the two problems. It incorporates random arrivals with multiple patient types and priorities as well as random appointment durations. We take into account the waiting time until the day of service as well as the idle time and overtime of medical resources on the day of service. We use approximate dynamic programming and determine advance schedules with stochastic appointment durations. We first extend the current literature by providing theoretical and numerical results for the case with multi-class, multi-priority patients and deterministic service times. We then adapt the model to incorporate stochastic service times and perform a comprehensive numerical analysis on a number of scenarios. We compare policies obtained from our models against benchmark policies used in practice. In addition, we present results based on a medium-size clinic in Ontario, Canada. Keywords: OR in health services, Patient scheduling, Markov decision processes, Approximate dynamic programming, Linear programming
\end{abstract}

\footnotetext{
${ }^{*}$ Corresponding author

Email addresses: asaure@uottawa.ca (Antoine Sauré), mbegen@ivey.uwo.ca (Mehmet A. Begen), patrick@telfer.uottawa.ca (Jonathan Patrick)
} 


\section{Introduction}

The large body of literature associated with patient scheduling can broadly be divided into two streams: appointment (or allocation) scheduling and advance scheduling. Advance scheduling refers to the allocation of future service capacity to demand as it arrives. It is most often done on a daily basis. Appointment scheduling, on the other hand, refers to the assignment of specific appointment times and resources to patients but only once all patients for a given service day have been identified. While there is a significant stream of literature regarding both types of scheduling problem, little work has been done that attempts to combine the two despite the potential of their being highly dependent on each other. A typical advance scheduling model will use resource utilization or overtime as a relevant performance metric. However, in the presence of stochastic service times, overtime is clearly dependent on the form of the appointment schedule. Conversely, the key input to an appointment scheduling problem is the number of patients to be served, which is precisely the output of an advance scheduling model. This interdependency of the two scheduling problems provides the impetus for this research that looks to determine the advantage of solving them as a single problem.

Advance scheduling problems typically assume that patients can be classified into multiple types according to their capacity requirements and urgency, that there is (at least) one resource that has a fixed regular-hour capacity, that there exists the possibility of using overtime or an alternative source of surge capacity, and that service durations are deterministic. The aim is to identify effective ways of allocating available service capacity to incoming appointment requests while either maximizing the service level (the number of patients booked within medically acceptable wait times) in a cost-effective manner or else maximizing revenue or throughput. Application areas include the scheduling of diagnostic tests such as MRIs (Schütz and Kolisch, 2012) or CT scans (Patrick et al., 2008) as well as radiation therapy treatments (Sauré et al., 2012). Papers in the area of advance scheduling mostly use dynamic programming, or approximate dynamic programming, due to the sequential nature of the scheduling decisions.

Appointment (or allocation) scheduling problems typically consider a single resource with high idle time and overtime costs. The objective is to determine appointment times (sequence) and appointment durations such that some combination of the costs associated with patient within-day waits, resource idle time and overtime is minimized. Appointment times are needed before the service day. The challenge comes from uncertain service times. There are many applications for appointment scheduling with two significant applications being surgery and physician appointment 
scheduling (Begen and Queyranne, 2011). Stochastic programming, queuing theory, simulation and simulation-optimization are commonly used methodologies to solve this type of problem.

In advance scheduling problems, the assumption of deterministic service times is largely made for convenience and in the general hope that, over time, average service times will work fairly well as an approximation. This assumption allows overtime or idle time to be easily calculated as the number of appointments booked on a given day times the appointment length minus the regular-hour capacity. In the absence of this assumption, the performance metrics of the advance schedule (namely overtime and idle time) are dependent on the appointment schedule being used. The purpose of this research is to test whether or not using average service times works well as an approximation.

To that end, we begin with the advance scheduling model provided in Patrick et al. (2008). Patients request service on a daily basis and are categorized based on urgency into multiple priority classes, each class with its own wait time target (medically acceptable wait time). The aim of the scheduler is to ensure that as many patients as possible are served within their wait time targets and with as little overtime as possible. The model penalizes scheduling a patient past his/her wait time target but does not prohibit it. Patrick et al. (2008) model this problem as a Markov Decision Process (MDP). However, the size of the state space and the corresponding action sets prohibit the determination of a solution via traditional methods. Instead, they resort to the linear programming approach to Approximate Dynamic Programming (ADP). In this approach, the value function in the MDP model is approximated by a function that is affine in the state variables and then the optimal approximation parameter values are determined using mathematical programming. The authors were able to determine the form of the optimal affine approximation under mild conditions on the availability of sufficient regular-hour and overtime capacity. This allowed for the characterization of the approximate optimal policy without having to solve an optimization model every time scheduling decisions were required. The result was an easy-to-implement heuristic policy that performs well in practice.

One of the limiting assumptions of Patrick et al. (2008) is that service times are homogeneous. The first contribution of this paper is to remove that assumption and allow for patient classification both by priority and by resource consumption. Initially, we maintain the assumption that resource consumption is deterministic. In this setting, we are able to show that the optimal affine approximation continues to have a predictable (though different) form and thus the approximate optimal policy can again be characterized and implemented without recourse to an optimization 
model. The proof of this theoretical result is provided in the online supplement.

The addition of stochastic service times significantly complicates the advance scheduling problem due to the fact that the computation of overtime now depends on the appointment schedule that defines the appointment time for each patient. For the purposes of this paper, we focus on the case where the only performance metric of interest for the appointment schedule is the overtime or idle time that results at the end of the service day. This assumption is reasonable in settings such as surgical scheduling where patient wait times on the day of service are not considered a major factor and where the possibility of resource idle time between appointments is removed by requiring patients to arrive well before their scheduled surgery times. The value of this simplifying assumption is that the calculation of the performance metrics does not depend on the sequencing of patients but only on their service times. Thus, the MDP model continues to evaluate the scheduling policy based on a combination of meeting the wait time target for each patient and minimizing the overtime and idle time at the end of the day of service. However, the calculation of the overtime or idle time is now based on a method described in Begen and Queyranne (2011) that determines the optimal appointment schedule as a function of known stochastic service time distributions.

We consider service times with discrete probability distributions. Thus, the expected cost on the service day (e.g., idle time or overtime cost) can be computed efficiently by using recursive equations for a given number of patients. Expected cost computations are fast (i.e., polynomial in the number of patients and in the largest service time) and can be done easily on an as-needed basis. Furthermore, these computations are flexible. We can either work with discrete probability distributions, if they are available, or samples of service durations. In the case of samples, we can work with correlated durations. Expected service-day costs are computed and incorporated into the MDP model.

In this paper, we describe a model setting that seeks to bridge advance and appointment scheduling problems. We obtain theoretical and numerical results for the advance scheduling problem with deterministic service times in the case of multi-class and multi-priority patients. Next, we incorporate stochastic service times into our model. We conduct a comprehensive analysis to compare the performance of the model with stochastic service durations to that of the model with deterministic service durations as well as against the performance of benchmark policies used in practice. In addition, we provide an extensive literature review of the advance and appointment scheduling problems. Last but not least, we present results based on a medium-size clinic in Ontario, Canada and quantify potential savings. 
The rest of the paper is organized as follows. In Section 2, we provide an exhaustive overview of the relevant literature. In Section 3, we describe an enhanced version of the MDP model presented in Patrick et al. (2008) that can be used to incorporate patient classes differentiated by both priority and resource consumption as well as stochastic service times. In Section 4, we first characterize the model with deterministic service times and then the model with stochastic service times. In particular, we state the theoretical results for the deterministic model with multiple service classes and show how stochastic service durations are incorporated. Then, in Section 5, we present extensive numerical results designed to determine the impact of incorporating stochastic service times and compare the performance of the resulting scheduling policies to that of benchmark policies used in practice. We also present results from a practical application based on data provided by a medium-size clinic. Finally, in Section 6, we provide a discussion and review our main conclusions.

\section{Related Literature}

Patient scheduling is complex due to its stochastic nature, the existence of multiple stages of care, scarce resources and multiple interdependencies between different stakeholders. As much as it is complex, it is also critical to have good and effective schedules to ensure that patients receive timely access to medical services in a cost-efficient manner. This is especially important now that health care costs and demand for medical services are on the rise and almost all countries are under constant pressure to improve health care efficiency while reducing costs.

Two important challenges in patient scheduling are the presence of random patient arrivals and the existence of random resource requirements. To address these challenges, researchers have mostly focused on one source of uncertainty at a time. Advance scheduling models deal with random patient arrivals whereas appointment scheduling models consider random service durations. Next, we provide an extensive literature survey on advance and appointment scheduling.

Appointment scheduling has been studied extensively over the last 50 years, starting with the well-known paper by Bailey (1952). This paper recommends booking two patients at the beginning of the day and patients equally spaced thereafter in order to avoid any idle time for doctors. Application areas of appointment scheduling include surgery scheduling (Denton et al., 2007; Begen and Queyranne, 2011) and physician appointment scheduling (Robinson and Chen, 2009; Kaandoorp and Koole, 2007). The main objective in appointment scheduling is to determine how much time to reserve for each appointment (Wang, 1993; Robinson and Chen, 2009; Denton and Gupta, 2003; Begen and Queyranne, 2011; Mancilla and Storer, 2012) and/or the number of 
appointments to book at each pre-determined time interval (Bosch et al., 1999; Kaandoorp and Koole, 2007; Muthuraman and Lawley, 2008; Chakraborty et al., 2010; Zeng et al., 2009; Cayirli et al., 2012) in order to minimize a weighted combination of the expected overtime and idle time of the resource (e.g., doctor's time or operating room hours) and the expected waiting time for patients. Almost all papers in the literature focus on the optimization of expected costs or rewards though there are some studies that consider other objectives. For example, Mittal et al. (2014) consider the worst-case scenario of the realized service times and Sang et al. (2017) the minimization of a percentile of the total cost.

In appointment scheduling, the total number of patients is assumed to be known in advance and planned appointment times are needed before any patient is served. The main challenge comes from uncertain appointment durations. Most papers in the literature use continuous probability distributions to describe service durations. However, there are some important advantages to considering discrete distributions instead (Begen and Queyranne, 2011). Discrete probability distributions enable efficient expected value computations and polynomial time algorithms for optimizing schedules. In addition, they can be easily used to incorporate no-shows and, to a certain extent, emergencies (Begen and Queyranne, 2011). There are also a few papers in the literature that discuss settings with partial, limited or no information on the service time distributions (Begen et al., 2012; Ge et al., 2013; Kong et al., 2013; Mak et al., 2014).

Although most studies assume that a fixed sequence of patients is given, and only determine an optimal schedule, the optimal sequencing of patients at the same time and in addition to determining the optimal appointment times is also considered in the literature. The problem, however, increases in complexity as the number of possible sequences grows quickly with the number of patients. Unsurprisingly, this problem is NP-hard (Mancilla and Storer, 2009). Some results exist on the optimal sequencing of two patients. For example, it has been demonstrated that scheduling patients in increasing order of service time variance is optimal (Weiss, 1990; Denton et al., 2007; Gupta, 2007). For a higher number of patients, the problem seems to be open and it is very unlikely that a universal optimal sequencing rule could be found unless some restrictions on the service time distributions and costs are imposed. For example, a recent paper by Guda et al. (2016) demonstrated that the shortest-variance-first rule is optimal for the single-machine earliness/tardiness problem if the earliness and tardiness cost parameters are the same for all the jobs and there is a dilation ordering of the processing times. The authors discuss the application of this rule to the appointment scheduling problem with sequencing in the case where idling is not 
allowed. Although an optimal sequencing rule for the general problem is not yet available, Denton et al. (2007) show that ordering patients by increasing service time variance works well. In addition, Chen and Robinson (2014) describe heuristic policies that work well in the presence of two classes of patients. Mak et al. (2014) show, using inventory approximations, that sequencing jobs based on increasing (standard deviation)/(overtime cost) ${ }^{\gamma}$, where $\gamma \in\{1,0.5\}$, performs better than simply ordering based on increasing variance.

While it is common to assume that both medical resources (e.g., doctors) and patients are punctual, there is substantial evidence suggesting that this assumption is often unwarranted. Furthermore, doctors' schedules can be interrupted by other, sometimes more urgent, tasks. Motivated by these considerations, there are a few papers that study the effect of unpunctuality and interruptions on the optimal appointment schedule (Klassen and Yoogalingam, 2009; Luo et al., 2012; Klassen and Yoogalingam, 2014; Samorani and LaGanga, 2015). There are also papers that consider the potential impact of patient no-shows, overbooking, and cancellations. Some of these papers resemble appointment scheduling, others come closer to advance scheduling.

The main techniques used for appointment scheduling are stochastic programming (Robinson and Chen, 2009; Denton and Gupta, 2003; Denton et al., 2007; Begen and Queyranne, 2011; Mancilla and Storer, 2012), the newsvendor approach (Weiss, 1990), queuing theory (Wang, 1993; Klassen and Rholeder, 1996; Kaandoorp and Koole, 2007), simulation (Santibáñez et al., 2007; White et al., 2011; Ma et al., 2016), and simulation-optimization (Klassen and Yoogalingam, 2008, 2009).

Advance scheduling differs from appointment scheduling in that arrivals are random while service durations are generally assumed to be deterministic (i.e., fixed). Advance scheduling thus deals with patient waiting times until the day of service (e.g., days until an MRI appointment or surgery), whereas appointment scheduling deals with costs incurred on the day of service (e.g., waiting time penalties, overtime and idle time costs). In advance scheduling one needs a booking (capacity allocation) policy to be used continuously, whereas in appointment scheduling one needs an appointment schedule before any patient is served.

Advance scheduling can be viewed as a stochastic capacity allocation problem in which the tradeoff between capacity utilization and waiting times or between revenue and service levels are modelled for different types of patients (Patrick et al., 2008; Erdelyi and Topaloglu, 2009; Dobson et al., 2011; Sauré et al., 2012; Truong, 2015). Alternatively, it can be considered a revenue management problem in which the main decision is to determine whether or not to accept an incoming service request (Schütz and Kolisch, 2012, 2013). While most studies in advance scheduling focus on a 
single resource (Patrick et al., 2008; Dobson et al., 2011), there are others that consider multiple resources (Gocgun and Ghate, 2012; Truong, 2015; Astaraky and Patrick, 2015). Other than in a few cases (Gupta and Denton, 2008; Wang and Gupta, 2011; Feldman et al., 2014), patient preferences have not been considered.

No-shows, in both appointment and advance scheduling, have received a significant amount of attention from researchers in the last few years (Liu, 2016; Tsai and Teng, 2014; Tang et al., 2014). High no-show rates can significantly paralyze a service and cause the double negative effect of low resource utilization and high waiting times. To overcome these negative outcomes, researchers have considered overbooking (Huang and Zuniga, 2012; LaGanga and Lawrence, 2012; Zacharias and Pindeo, 2014), studied the relationship between panel size and no-show rates (Green and Savin, 2008; Liu, 2016), and examined the impact of open-access policies (Robinson and Chen, 2010; Patrick, 2012). Open-access policies are also known as "same-day" policies. Patients call on the day of their appointments or only a few days before. There are also studies that consider cancellations (Liu et al., 2009; Schütz and Kolisch, 2012) and daily no-show estimates (Samorani and LaGanga, 2015).

Papers in the area of advance scheduling mostly use dynamic programming or approximate dynamic programming due to the stochastic nature of the appointment request arrivals and the sequential nature of the decision process (Patrick et al., 2008; Sauré et al., 2012; Schütz and Kolisch, 2012, 2013; Sauré et al., 2015; Truong, 2015). Some of the objectives considered in these studies are: maximizing the number of patients booked within their medically acceptable wait times (Patrick et al., 2008; Sauré et al., 2012; Sauré et al., 2015), maximizing revenue (Gupta and Denton, 2008; Schütz and Kolisch, 2013), improving resource utilization (Santibáñez et al., 2009), satisfying specific appointment date windows (Gocgun and Puterman, 2014), taking patient preferences into account (Gupta and Denton, 2008; Wang and Gupta, 2011; Feldman et al., 2014), and reducing wait times (Green et al., 2006). Application areas of advance scheduling include the scheduling of diagnostic tests such as MRI/CT scans (Green et al., 2006; Patrick et al., 2008; Schütz and Kolisch, 2012), radiation therapy treatment scheduling (Sauré et al., 2012), primary care clinics (Green and Savin, 2008; Dobson et al., 2011; Liu, 2016), and surgical scheduling (Astaraky and Patrick, 2015).

To the best of our knowledge, most related to our work are the papers of Muthuraman and Lawley (2008), Zeng et al. (2009), Chakraborty et al. (2010), and Erdogan and Denton (2013). In Muthuraman and Lawley (2008), the authors consider an advance scheduling problem with noshows and determine the number of appointments per day required to maximize revenue. They 
assume appointments slots of fixed length and exponentially distributed service times. Zeng et al. (2009) and Chakraborty et al. (2010) extend Muthuraman and Lawley (2008)'s work to the case of heterogeneous no-show probabilities and general service time distributions, respectively. To some degree, these three papers combine advance scheduling with appointment scheduling. However, the authors assume that appointment durations are fixed, of equal length, and determined exogenously. Except for no-show rates, patients are homogeneous (i.e., same service time and priority) and the admission decisions are made at the time of the booking requests. In Erdogan and Denton (2013), the authors use stochastic programming to formulate a dynamic appointment scheduling problem with uncertain demand and homogeneous patients. The maximum number of patients that can be scheduled is known and each appointment request is probabilistic given the state of the previous one. The authors only consider single-period models and provide a conceptual multi-stage stochastic programming formulation for the case of probabilistic appointment requests. They provide some structural properties and numerical results for the case of deterministic patient arrivals with noshows. None of these four papers attempts to characterize the optimal solution. Except for these papers, we are not aware of any other studies that combine both random patient arrivals and random service times in the context of appointment scheduling.

In this paper, we develop a framework that seeks to bridge advance and appointment scheduling problems. We consider random appointment requests coming from multiple types of patients as well as random appointment durations. Patients are classified on the basis of resource consumption and priority (given by urgency level or maximum recommended wait time). We take into account the wait until the day of service as well as the medical resource (e.g., operating room or surgeon) idle time and overtime at the end of the day of service. Patients' waiting within the day of service is not considered.

\section{An MDP Formulation for the Patient Scheduling Problem}

In this section, we formulate a discounted infinite-horizon MDP model for the problem under study. We assume that demand for service has been broken down into $I$ priority classes and $J$ service classes. Service classes are differentiated by the service time probability distribution or by the mean service time in the deterministic case. For notational simplicity, we let $[A]=\{1, \ldots, A\}$. We denote a vector by bolding it, such as $\mathbf{s}$. 


\subsection{Decision Epochs and the Booking Horizon}

We consider a system that has a capacity of $C^{R}$ regular time units and $C^{O T}$ overtime units per day. At a specific point of time every day, referred to as the decision epoch, the scheduler observes the number of booked appointments from each service class on each future day over an $N$-day booking horizon and the number of cases in each priority class-service class pairing waiting to be scheduled. The booking horizon is defined by the maximum number of days in advance that the scheduler is allowed to schedule patients.

We assume that the number of patients scheduled into a given service day is known prior to the start of the day. This, for example, reflects a hospital that chooses to reserve capacity for emergency surgeries rather than impinge on the elective surgical schedule. Our model is complicated by the fact that the booking horizon is not static but rolling. Thus, day $n$ at the current decision epoch becomes day $n-1$ at the subsequent decision epoch. Since no patient is scheduled more than $N$ days in advance, at the beginning of each decision epoch, the $N^{\text {th }}$ day has no appointments booked.

\subsection{The State Space}

As mentioned above, we assume that demand for service is broken down into multiple priority classes, based on urgency, and multiple service classes, based on the probability distribution of the length of service. Our state takes the form $\mathbf{s}=(\mathbf{x}, \mathbf{y})$, where $x_{j n}$ is the number of patients from service class $j$ already booked on day $n$ and $y_{i j}$ is the number of priority $i$ patients from service class $j$ waiting to be booked. The state space needs to capture the number of patients of each service class booked into each day of the booking horizon as the service class mix will play a key role in determining overtime and idle time costs and the optimal appointment schedule, particularly in the scenario with stochastic service times. In contrast, prioritization does not need to be tracked once a patient has been scheduled to a specific day as the value of any late booking penalty is determined at the time of booking.

\subsection{The Potential Action Sets}

The scheduler's task is to decide at each decision epoch on which day to schedule each of the patients waiting to be booked. Thus, a vector of possible actions can be written as a, where $a_{i j n}$ is the number of priority $i$ patients from service class $j$ to book on day $n$. To accommodate the potential for overtime, bookings are allowed to exceed the daily regular-hour capacity up to a limit $C^{O T}$ of overtime units. To be valid, the number of bookings cannot exceed the number of patients waiting, 


$$
\sum_{n=1}^{N} a_{i j n} \leq y_{i j} \quad \forall(i, j) \in[I] \times[J],
$$

and the overtime capacity cannot be exceeded,

$$
\sum_{j=1}^{J} \mu_{j}\left(x_{j n}+\sum_{i=1}^{I} a_{i j n}\right) \leq C^{R}+C^{O T} \quad \forall n \in[N],
$$

where $\mu_{j}$ is the number of time units required by a patient of service class $j$. For the stochastic case, $\mu_{j}$ can represent either the average service time or else some percentile of the service time depending on how conservative the scheduler wants to be. Finally, all actions are constrained to be positive and integer,

$$
\mathbf{a} \in \mathbb{Z}_{[I] \times[J] \times[N]}
$$

We define the action set $A(\mathbf{s})$, for any given state $\mathbf{s}$, as the set of actions a satisfying equations (1) to $(3)$.

\subsection{Transition Probabilities}

Once decisions are made, the only stochastic element in the transition to the next state of the system is due to the number of new appointment requests in each priority class-service class pairing. Demand that is not booked today re-appears in tomorrow's demand. If the number of new patient arrivals is represented by $\mathbf{y}^{\prime}$, then

$$
\begin{array}{ll}
x_{j n} \rightarrow x_{j, n+1}+\sum_{i=1}^{I} a_{i j, n+1} & \forall(j, n) \in[J] \times[N], \\
y_{i j} \rightarrow y_{i j}^{\prime}+y_{i j}-\sum_{n=1}^{N} a_{i j n} & \forall(i, j) \in[I] \times[J],
\end{array}
$$

where $x_{i j, N+1}=a_{i j, N+1}=0$. We assume demand from each patient priority class-service class pairing is independent and that each day's demand is independent as well. Expected demand values are denoted by $\lambda_{i j}$. 


\subsection{Immediate Costs}

The cost associated with a given state-action pair derives from three sources: penalties associated with booking patients beyond the priority-specific wait time targets, a cost associated with the day of service (overtime or idle time), and penalties associated with delaying the booking decision for some of the waiting demand. We write the immediate cost function as

$$
c(\mathbf{s}, \mathbf{a})=\sum_{(i, n) \in[I] \times[N]} f^{W T}(i, n) \sum_{j \in[J]} a_{i j n}+f^{A S}(\mathbf{s}, \mathbf{a})+\sum_{(i, j) \in[I] \times[J]} f^{D}(i)\left(y_{i j}-\sum_{n \in[N]} a_{i j n}\right),
$$

where $f^{W T}(i, n)$ is the penalty associated with booking a priority $i$ patient on day $n$ and $f^{A S}(\mathbf{s}, \mathbf{a})$ is the cost associated with the appointment schedule. The latter could consist of a combination of within-day wait times of patients as well as idle time and overtime at the end of the day. This cost will be discussed further in Section 4 as we look at both the deterministic and the stochastic version of the model separately. $f^{D}(i)$ is the penalty associated with delaying a priority $i$ patient's booking one day. We represent the wait time target for priority $i$ by $T(i)$. The choice of $f^{W T}(i, n)$, though arbitrary, should consider certain characteristics. It is clearly reasonable to assume that it should be decreasing in $i$ and zero if $n \leq T(i)$. Furthermore, it would seem advisable to ensure that the penalty associated with delaying a patient's booking $k$ days and then booking him/her within the corresponding wait time target should be equal to the penalty associated with booking the patient $k$ days late initially. Thus, a natural form for the wait time penalty is

$$
f^{W T}(i, n)= \begin{cases}\sum_{k=1}^{n-T(i)} \gamma^{k-1} f^{D}(i), & \text { for all } n>T(i) \\ 0, & \text { otherwise }\end{cases}
$$

where $\gamma$ is the daily discount factor.

The immediate cost function $c(\mathbf{s}, \mathbf{a})$ explicitly balances the cost to the patients in wait times and the cost to the system in having to resort to overtime (or having idle time). The scheduler's role is to maintain reasonable patient wait times in a cost-effective manner.

\subsection{The Structure of the Bellman Equation}

The value function $v$ of the MDP specifies the minimum expected discounted cost over the infinite horizon for each state and satisfies the following optimality equations: 


$$
\begin{aligned}
v(\mathbf{s})=\min _{\mathbf{a} \in A(\mathbf{s})}\left\{c(\mathbf{s}, \mathbf{a})+\gamma \sum_{\mathbf{y}^{\prime} \in D} p\left(\mathbf{y}^{\prime}\right) v\left(x_{12}+\sum_{i=1}^{I} a_{i 12}, \ldots, x_{J N}+\sum_{i=1}^{I} a_{i J N}, 0\right.\right. \\
\left.\left.y_{11}^{\prime}+y_{11}-\sum_{n=1}^{N} a_{11 n}, \ldots, y_{I J}^{\prime}+y_{I J}-\sum_{n=1}^{N} a_{I J n}\right)\right\} \forall \mathbf{s} \in S,
\end{aligned}
$$

where $D$ is the set of all possible incoming demand streams and $p\left(\mathbf{y}^{\prime}\right)$ is the probability of a vector $\mathbf{y}^{\prime}$ of new demand.

\section{Deterministic and Stochastic Models}

The formulation presented in the previous section provides a comprehensive model that incorporates multiple patient classes with a flexible function $f^{A S}(\mathbf{s}, \mathbf{a})$ for within-day costs. In this section, we show how $f^{A S}(\mathbf{s}, \mathbf{a})$ can be computed for the deterministic case and the stochastic case and describe an ADP version of the model.

\subsection{Deterministic Model}

In the deterministic case, the within-day costs are reduced to overtime and idle time costs and can be computed as

$$
f^{A S}(\mathbf{s}, \mathbf{a})=h\left[\sum_{j=1}^{J} \mu_{j}\left(x_{j 1}+\sum_{i=1}^{I} a_{i j 1}\right)-C^{R}\right]^{+}+u\left[C^{R}-\sum_{j=1}^{J} \mu_{j}\left(x_{j 1}+\sum_{i=1}^{I} a_{i j 1}\right)\right]^{+}
$$

where $h$ is the overtime cost per time unit, $u$ is the idle time cost per time unit, and $[a]^{+}=\max (0, a)$.

Even in the deterministic case, the size of the state space and the size of the corresponding action sets require that we approximately solve our model via ADP. To that end, we assume that the value function in our formulation can be adequately represented by an affine approximation architecture in the form:

$$
V(\mathbf{s})=V_{0}+\sum_{\substack{(j, n) \in \\[J] \times[N]}} V_{j, n} x_{j n}+\sum_{\substack{(i, j) \in \in \\[I] \times[J]}} W_{i j} y_{i j} \quad \forall \mathbf{s} \in S \quad \mathbf{V}, \mathbf{W} \geq 0, V_{0} \in \mathbb{R} .
$$

There are two main options in seeking to solve an MDP model using ADP. Simulation-based ADP iteratively produces simulated runs of the model to approximate the value function at a subset of initial states while using a form of least squares regression or a recursive update function 
to converge on a good approximation (e.g., see Sauré et al., 2015). The other option is to transform the MDP model into an equivalent linear program and then substitute into it the value function approximation of choice. This is the approach taken here and in Patrick et al. (2008).

Once the approximation given in Equation (7) is substituted into the LP formulation of the discounted MDP, we get the following approximate linear program (ALP):

$$
\min _{\substack{\mathbf{v}, \mathbf{W} \geq 0, V_{0} \in \mathbb{R}}} \sum_{\mathbf{s} \in S} \alpha(\mathbf{s})\left(V_{0}+\sum_{\substack{(j, n) \in \\[J] \times[N]}} V_{j n} x_{j n}+\sum_{\substack{(i, j) \in \\[I] \times[J]}} W_{i j} y_{i j}\right)
$$

subject to

$$
\begin{aligned}
& (1-\gamma) V_{0}+\sum_{\substack{(j, n) \in \\
[J] \times[N]}}\left(x_{j n}-\gamma x_{j, n+1}-\gamma \sum_{i \in[I]} a_{i j, n+1}\right) V_{j n}+ \\
& \sum_{\substack{(i, j) \in \\
[I] \times[J]}}\left((1-\gamma) y_{i j}+\gamma \sum_{n \in[N]} a_{i j n}-\gamma E\left[Y_{i j}\right]\right) W_{i j} \geq c(\mathbf{s}, \mathbf{a}) \quad \forall(\mathbf{s}, \mathbf{a}) \in S \times A(\mathbf{s}),
\end{aligned}
$$

where $\alpha$ is any positive-valued vector. In traditional MDP theory, the choice of $\alpha$ is arbitrary as any $\alpha>0$ will lead to the same solution. This turns out not to be the case when using ADP as the choice of $\alpha$ plays a key role in the solution. The value of $\alpha$ is best interpreted as the probability distribution over the initial state of the system.

While the ALP remains intractable due to the existence of a constraint for every state-action pair, its dual can be solved via column generation. The dual of the ALP can be written as:

$$
\max _{\mathbf{X} \geq 0} \sum_{\substack{(\mathbf{s}, \mathbf{a}) \in \\ S \times A(\mathbf{s})}} X(\mathbf{s}, \mathbf{a}) c(\mathbf{s}, \mathbf{a})
$$

subject to

$$
\begin{gathered}
(1-\gamma) \sum_{\substack{(\mathbf{s}, \mathbf{a}) \in \\
S \times A(\mathbf{s})}} X(\mathbf{s}, \mathbf{a})=1, \\
\sum_{\substack{(\mathbf{s}, \mathbf{a}) \in \\
S \times A(\mathbf{s})}} X(\mathbf{s}, \mathbf{a})\left(x_{j n}-\gamma x_{j, n+1}-\gamma \sum_{i=1}^{I} a_{i j, n+1}\right) \geq E_{\alpha}\left[X_{j n}\right] \quad \forall(j, n) \in[J] \times[N], \\
\sum_{\substack{\mathbf{s}, \mathbf{a} \in \\
S \times A(\mathbf{s})}} X(\mathbf{s}, \mathbf{a})\left((1-\gamma) y_{i j}+\gamma \sum_{n \in[N]} a_{i j n}-\gamma E\left[Y_{i j}\right]\right) \geq E_{\alpha}\left[Y_{i j}\right] \quad \forall(i, j) \in[I] \times[J] .
\end{gathered}
$$

The dual variable $X(\mathbf{s}, \mathbf{a})$ can be interpreted as the frequency of taking action a when in state s. Following our earlier comment regarding $\alpha$, one can interpret $E_{\alpha}\left[X_{j n}\right]$ as the expected number of 
patients of type $j$ booked into day $n$ and $E_{\alpha}\left[Y_{i j}\right]$ as the expected number of new arrivals of priority class $i$ and service class $j$ both associated with the initial state of the system.

Once the optimal value function approximation has been determined then the approximate optimal decision policy is derived by determining the arg min of the optimality equation given in Equation (5), with the optimal approximation inserted in the place of the value function. If the regular-hour capacity on day 1 is full, that is $\sum_{j \in[J]} \mu_{j}\left(x_{j 1}+\sum_{i \in[I]} a_{i j 1}\right)-C^{R}>0$, then $\mathbf{a}^{*}$ is given by

$$
\min _{\mathbf{a} \in A(\mathbf{s})}\left[\mathcal{C}+\sum_{\substack{(i, j) \in \\[I] \times[J]}}\left(h \mu_{j}-f^{D}(i)-\gamma W_{i j}^{*}\right) a_{i j 1}+\sum_{\substack{(i, j) \in \\[I] \times[J]}} \sum_{n=2}^{N}\left(f^{W T}(i, n)+\gamma V_{j, n-1}^{*}-f^{D}(i)-\gamma W_{i j}^{*}\right) a_{i j n}\right] .
$$

Otherwise, if there is regular-hour capacity available on day 1 , then $\mathbf{a}^{*}$ is given by

$$
\min _{\mathbf{a} \in A(\mathbf{s})}\left[\mathcal{C}+\sum_{\substack{(i, j) \in \in \\[I] \times[J]}}\left(-u \mu_{j}-f^{D}(i)-\gamma W_{i j}^{*}\right) a_{i j 1}+\sum_{\substack{(i, j) \in \in \\[I] \times[J]}} \sum_{n=2}^{N}\left(f^{W T}(i, n)+\gamma V_{j, n-1}^{*}-f^{D}(i)-\gamma W_{i j}^{*}\right) a_{i j n}\right],
$$

where $\mathcal{C}$ is a constant independent of the action taken. The coefficients in equations (10) and (11) have a nice intuitive explanation. In the case where day 1's capacity is full, the bookings on day 1 trade off the cost associated with overtime for the cost of delaying a booking and having an additional patient in the wait list tomorrow. In the case where there is excess capacity on day 1 , there is only a benefit to making use of that capacity as it reduces the idle time cost. Thus, the coefficients for bookings on day 1 are negative. The bookings on day $n>1$ trade off a potential wait time penalty plus the cost of having less available capacity on day $n-1$ tomorrow for the cost of delaying a booking and having an additional patient in the wait list tomorrow.

Somewhat surprisingly but as a natural extension to Patrick et al. (2008), the form of the optimal affine value function approximation can be proven under certain conditions on the cost values and the available capacity as outlined in Theorem 1. The wait time targets for each priority class, $T(i)$, are assumed to increase with $i$ as a high priority patient is, by definition, a patient who must be served sooner. We define the indicator function $I(\cdot)$ as

$$
I(X>x)= \begin{cases}1, & X>x \\ 0, & \text { otherwise. }\end{cases}
$$

to ease the presentation. 
Theorem 1. Assuming that $T(i)$ is non-decreasing in $i$, that the wait time penalties are nondecreasing in $n$ and non-increasing in $i$, and that the following conditions are satisfied:

$$
\begin{gathered}
f^{D}(i)>\left(\gamma^{T(i)-1}-\gamma^{T(i)}\right) \mu_{j} h \quad \forall(i, j) \in[I] \times[J] \\
\sum_{j \in J} \mu_{j}\left[\sum_{i=1}^{I} \frac{\gamma^{T(i)-1} I(T(i)>1) \lambda_{i j}}{1-\gamma}+\sum_{m=1}^{N} \gamma^{m-1} E_{\alpha}\left[X_{j m}\right]\right]>\frac{C^{R}}{1-\gamma} \\
\sum_{j \in J} \mu_{j}\left[\sum_{i=1}^{I} \frac{\gamma^{T(i)-n} I(T(i)>n) \lambda_{i j}}{1-\gamma}+\sum_{m=n}^{N} \gamma^{m-n} E_{\alpha}\left[X_{j m}\right]\right]<\frac{C^{R}+C^{O T}}{1-\gamma} \quad \forall n \in[N]
\end{gathered}
$$

Then, the optimal affine value function approximation for the discounted MDP will be given by

$$
\begin{aligned}
V_{j n}^{*} & = \begin{cases}\mu_{j} h, & n=1 ; \\
\gamma V_{j, n-1}^{*}, & 2 \leq n \leq N-1 ; \\
0, & n=N .\end{cases} \\
W_{i j}^{*} & = \begin{cases}V_{j T(i)}^{*}, & \lambda_{i j}>0 ; \\
0, & \lambda_{i j}=0 .\end{cases} \\
V_{0}^{*} & =\frac{1}{1-\gamma}\left(\begin{array}{c}
\sum_{\substack{(i, j) \in \\
[I] \times[J]}} \gamma^{T(i)} \mu_{j} h E\left[Y_{i j}\right]+h C^{R}
\end{array}\right) .
\end{aligned}
$$

Conditions (12) to (14) have a nice intuitive appeal. Condition (12) ensures that the cost of delaying a booking decision is greater than the difference between serving a patient through overtime $T(i)$ days from now versus $T(i)-1$ days from now. If this condition is not satisfied then the optimal action is simply not to book patients unless there is regular capacity available. In other words, the cost of overtime is prohibitive. If $\alpha$ is viewed as a probability distribution on the initial state of the system then the left-hand side of Condition (13) can be seen as the present value of the expected demand over the infinite horizon plus the initial bookings (all in time units). This is required to be greater than the present value of the regular-hour capacity over the infinite horizon. Satisfying this condition ensures that there is in fact a congestion problem. If it is not satisfied then a first-come, first-served booking policy would work equally well as regular-hour capacity would be sufficient. Finally, Condition (14) ensures that, for any given day $n$, the present value of the expected future demand with wait time targets greater than $n$ over the infinite horizon plus the initial bookings on days that are greater than $n$ (all in time units) are less than the present value 
of the combined overtime and regular-hour capacity. In other words, it guarantees that there is sufficient overtime capacity to deal with the fluctuations in demand. This condition is particularly appealing as it provides a capacity requirement to ensure that the resulting booking policy will work well in practice. The proof of Theorem 1 is provided in the online supplement.

\subsection{Stochastic Model}

As mentioned earlier in this paper, we only consider the end-of-day overtime and idle time costs to determine the within-day cost. Thus, in the stochastic case, we can compute $f^{A S}(\mathbf{s}, \mathbf{a})$ as

$$
f^{A S}(\mathbf{s}, \mathbf{a})=h\left[D(\mathbf{s}, \mathbf{a})-C^{R}\right]^{+}+u\left[C^{R}-D(\mathbf{s}, \mathbf{a})\right]^{+},
$$

where $D(\mathbf{s}, \mathbf{a})$ is the sum of the durations of all the appointments booked on day 1 after taking action a in state $\mathbf{s}$. That is $D(\mathbf{s}, \mathbf{a})=\sum_{k=1}^{K(\mathbf{s}, \mathbf{a})} d_{k}$, where $d_{k}$ is the duration of appointment $k$ and $K(\mathbf{s}, \mathbf{a})=\sum_{j=1}^{J}\left(x_{j 1}+\sum_{i=1}^{I} a_{i j 1}\right)$ is the total number of appointments booked on day 1 . We assume $d_{k}$ to be bounded from above and below so that $\underline{d}_{k} \leq d_{k} \leq \bar{d}_{k} \forall k$.

We use discrete probability distributions to model appointment durations and the algorithm in Begen and Queyranne (2011) to compute the probability distribution of $D(\mathbf{s}, \mathbf{a})$ and the expected value of $f^{A S}(\mathbf{s}, \mathbf{a}), \forall(\mathbf{s}, \mathbf{a}) \in S \times A(\mathbf{s})$, when the service time distributions are known and independent. When the probability distributions are unknown and only samples of $D(\mathbf{s}, \mathbf{a})$ are available, then we can use the approach in Begen et al. (2012) instead. In this case, we do not require any independence assumption as we can work with correlated appointment durations as well. It is also important to note that, in the case of known probability distributions, there are other methods for computing the probability distribution of $D(\mathbf{s}, \mathbf{a})$ (e.g., see Drew et al., 2008).

To compute the expected value of $f^{A S}(\mathbf{s}, \mathbf{a})$ when the duration of appointment $k$ follows a known discrete probability distribution $\operatorname{Pr}\left\{d_{k}=m\right\}, \underline{d}_{k} \leq m \leq \bar{d}_{k}$, we first need to determine the probability distribution of $D(\mathbf{s}, \mathbf{a})$. If we let $D_{a b}=\sum_{t=a}^{b} d_{t} \forall a, b$ then this is equivalent to computing the probability distribution of $D_{1 K(\mathbf{s}, \mathbf{a})}$. To that end, we start by computing the probability distribution of $D_{11}=d_{1}$, then we find the probability distribution of $D_{12}=d_{1}+d_{2}, D_{1 k}=D_{1(k-1)}+d_{k}$, $\ldots, D_{1 K}=D_{1(K-1)}+d_{K}$, recursively. For example, 


$$
\begin{array}{rlr}
\operatorname{Pr}\left\{D_{1 b}=k\right\} & =\operatorname{Pr}\left\{D_{1(b-1)}+d_{b}=k\right\} & \forall k \\
& =\sum_{m=\underline{d}_{k}} \operatorname{Pr}\left\{D_{1(b-1)}=k-m, d_{b}=m\right\} & \forall k \\
& =\sum_{m=\underline{d}_{k}}^{\bar{d}_{k}} \operatorname{Pr}\left\{D_{1(b-1)}=k-m \mid d_{b}=m\right\} \operatorname{Pr}\left\{d_{b}=m\right\} & \forall k \\
& =\sum_{m=\underline{d}_{k}} \operatorname{Pr}\left\{D_{1(b-1)}=k-m\right\} \operatorname{Pr}\left\{d_{b}=m\right\} \quad \text { (independence) } & \forall k
\end{array}
$$

We must repeat these steps for all possible values of $b$ and $k$ as indicated in the pseudo-code presented in the online supplement. These computations are polynomial in $K$ and $\bar{d}_{\max }$, where $\bar{d}_{\max }=\max \left\{\bar{d}_{1}, \bar{d}_{2}, \ldots, \bar{d}_{K}\right\}$.

Once we have the probability distribution of $D(\mathbf{s}, \mathbf{a})$, that is $\operatorname{Pr}\left\{D_{1 K(\mathbf{s}, \mathbf{a})}=m\right\} \forall m$, we can compute the expectation of $f^{A S}(\mathbf{s}, \mathbf{a})$ by using Equation (18) as follows:

$$
\sum_{k \leq C^{R}} u\left(C^{R}-k\right) \operatorname{Pr}\left\{D_{1 K}=k\right\}+\sum_{k>C^{R}} h\left(k-C^{R}\right) \operatorname{Pr}\left\{D_{1 K}=k\right\}
$$

As mentioned above, we can also use a sampling approach to compute the expected value of $f^{A S}(\mathbf{s}, \mathbf{a})$ when the service time probabilities are unknown. Let us assume that we have access to $N$ samples $\left\{D_{t}\right\}_{t=1}^{N}$ of $D(\mathbf{s}, \mathbf{a})$, then the expected within-day cost can be computed in polynomial time as

$$
\frac{1}{N}\left[\sum_{t=1}^{N} h\left(D_{t}-C^{R}\right)^{+}+u\left(C^{R}-D_{t}\right)^{+}\right] .
$$

After the expected within-day cost is computed for all possible patient service class combinations on day 1, we can use the same ADP approach as for the deterministic case. The implementation of the column generation algorithm and the optimization model employed to identify the approximate optimal actions was performed in GAMS 24.2 with CPLEX 12.6 as the solver. The algorithm used to compute the expected within-day costs was implemented in Java.

\section{Numerical Results}

To explore the impact of incorporating stochastic service times into the MDP model described in Section 3, we performed an extensive numerical analysis under a number of scenarios. Scenarios 
are categorized into four problem settings. These settings were designed to reflect common observed practices in health systems as well as situations where stochastic service times might reasonably be expected to have a higher impact. Next, we compare the performance of the appointment scheduling policies suggested by the proposed approach, that are greedy with respect to the final affine value function approximations, to that of the two other policies described below:

- First Available Slot (FAS): Patients are booked as soon as possible, in increasing priority class and service class order, according to their expected service times and the available regular-hour capacity. This policy resorts to overtime only when there is no available regular-hour capacity within the booking horizon. Overtime is then booked starting with day 1 and working up to day $N$.

- Myopic (M): Patients are booked as soon as possible, in increasing priority class and service class order, according to their expected service times and the available regular-hour capacity. Unlike the previous policy, this policy resorts to overtime for patients of type $i$ only when there is no available regular-hour capacity within the first $n_{i}$ days of the booking horizon, where $n_{i}=\max \left\{n: f^{W T}(i, n)<h\right\}$. Overtime is then booked starting with day 1 and working up to day $N$.

These two policies are the most common representations of actual scheduling practices reported in the literature and observed in practice. The approximate optimal policies obtained from the deterministic and the stochastic version of our model are denoted by AOPD and AOPS, respectively. The AOPD policy can be viewed as a revised version of the policy described in Patrick et al. (2008) since our model extends the MDP model in Patrick et al. (2008) to include multiple service classes.

Although the MDP model described in Section 3 is formulated in terms of the expected discounted cost (DC) over the infinite horizon, the different patient scheduling policies are also compared in terms of mean daily average cost (AC), mean daily average capacity utilization (ACU), mean average time to first available appointment slot (ATFAS), mean average wait times (AWTs) and mean service levels (SLs). The ATFAS is computed for each service class as the time to the first day for which the available regular-hour capacity exceeds the corresponding expected service time. The SL is computed for each priority class as the percentage of patients booked within the corresponding wait time target.

To compare the performance of the different patient scheduling policies we ran 100 replications of a simulation of the scheduling process with a length of 1500 days and a warm-up period of 
1000 days. We used a computer with a $3.00 \mathrm{GHz}$ Quad Core CPU and 16 GB of RAM for all the numerical experiments in this paper. The simulation model was implemented using GAMS Java Application Program Interface (API) with common patient arrivals and service durations.

\subsection{Problem Setting 1: Base Case}

We first consider a system with a regular-hour capacity of 18 appointment slots per day. The overtime capacity is set at 9 appointment slots per day. The system divides demand into three priority classes, with wait time targets of 4, 8 and 12 days, and three service classes, with expected service times of 2, 3 and 4 appointment slots. Demand from each priority class-service class combination is assumed to be Poisson with the means given in Table 1. The total expected demand is equal to the regular-hour capacity. We consider service time probability distributions that are geometric, negative binomial, Poisson and uniform. The overtime cost is 100 per appointment slot, the idle time cost is 50 per appointment slot, the postponement penalties are 20,10 and 5 per patient for each priority class, and the discount factor is 0.99 . We assume that no patient is scheduled more than 12 days in advance.

Table 1: Arrival rate for each priority class-service class combination for Problem Setting 1 (Base Case).

\begin{tabular}{ccccc}
\hline \multirow{2}{*}{ Priority Class } & \multicolumn{4}{c}{ Service Class } \\
& 1 & 2 & 3 & Total \\
\hline 1 & 1.0 & 1.0 & 1.0 & 3.0 \\
2 & 1.0 & 1.0 & - & 2.0 \\
3 & - & - & 1.0 & 1.0 \\
\hline Total & 2.0 & 2.0 & 2.0 & 6.0 \\
\hline
\end{tabular}

The simulation results are summarized in Table 2 for initial states generated using the FAS policy and negative binomial service time distributions. The results for initial states generated using the M policy, included in the online supplement, are similar, suggesting that the simulation outcomes could be independent of the warm-up policy. Table 3 shows the mean discounted cost for the different discrete service time distributions.

Although the AOPS policy tended to outperform the AOPD policy in the simulation, the difference in the mean discounted cost was minor (around 1\%) even for service time distributions with high variance. On the other hand, both ADP policies outperform the FAS and M policies by significant margins. In particular, for the higher priority patients, the service levels and average wait times provided by the ADP policies are dramatically better. The ability of the booking policy obtained from the deterministic model to almost match the performance of the one coming from the 
Table 2: Summary of the simulation results for Problem Setting 1 (Base Case). The bold font indicates the policy (policies) that provides (provide) the best mean performance for each metric in a statistical sense $(\alpha=0.05)$

\begin{tabular}{cccccc}
\hline \multirow{2}{*}{ Metric } & $\begin{array}{c}\text { Priority/Service } \\
\text { Class }\end{array}$ & AOPD & AOPS & FAS & M \\
\hline DC & - & $34054 \pm 667$ & $\mathbf{3 3 8 0 2} \pm \mathbf{6 9 9}$ & $58312 \pm 2482$ & $46793 \pm 1422$ \\
\hline AC & - & $314.46 \pm 1.83$ & $\mathbf{3 1 2 . 1 3} \pm \mathbf{1 . 7 6}$ & $589.87 \pm 13.82$ & $439.34 \pm 7.82$ \\
\hline ACU & - & $18.05 \pm 0.05$ & $18.05 \pm 0.05$ & $\mathbf{1 8 . 0 0} \pm \mathbf{0 . 0 5}$ & $18.03 \pm 0.05$ \\
\hline \multirow{2}{*}{ ATFAS } & 1 & $\mathbf{1 . 2 6} \pm \mathbf{0 . 0 1}$ & $1.28 \pm 0.01$ & $2.27 \pm 0.02$ & $2.19 \pm 0.02$ \\
& 2 & $\mathbf{1 . 3 0} \pm \mathbf{0 . 0 1}$ & $1.33 \pm 0.01$ & $2.82 \pm 0.03$ & $2.66 \pm 0.03$ \\
& 3 & $\mathbf{1 . 3 5} \pm \mathbf{0 . 0 1}$ & $1.38 \pm 0.01$ & $3.60 \pm 0.05$ & $3.29 \pm 0.04$ \\
\hline \multirow{2}{*}{$\mathrm{AWT}$} & 1 & $\mathbf{2 . 0 2} \pm \mathbf{0 . 0 2}$ & $2.18 \pm 0.02$ & $8.72 \pm 0.19$ & $6.27 \pm 0.11$ \\
& 2 & $\mathbf{5 . 5 1} \pm \mathbf{0 . 0 4}$ & $\mathbf{5 . 5 0} \pm \mathbf{0 . 0 4}$ & $8.85 \pm 0.18$ & $7.02 \pm 0.13$ \\
& 3 & $9.49 \pm 0.05$ & $9.64 \pm 0.05$ & $8.68 \pm 0.15$ & $\mathbf{7 . 4 5} \pm \mathbf{0 . 1 3}$ \\
\hline \multirow{2}{*}{$\mathrm{SL}$} & 1 & $\mathbf{9 9 . 8 9} \pm \mathbf{0 . 0 3}$ & $99.84 \pm 0.04$ & $9.64 \pm 1.73$ & $23.32 \pm 1.84$ \\
& 2 & $\mathbf{1 0 0 . 0 0} \pm \mathbf{0 . 0 0}$ & $\mathbf{1 0 0 . 0 0} \pm \mathbf{0 . 0 0}$ & $36.22 \pm 2.77$ & $67.55 \pm 1.85$ \\
& 3 & $\mathbf{1 0 0 . 0 0} \pm \mathbf{0 . 0 0}$ & $\mathbf{1 0 0 . 0 0} \pm \mathbf{0 . 0 0}$ & $\mathbf{1 0 0 . 0 0} \pm \mathbf{0 . 0 0}$ & $\mathbf{1 0 0 . 0 0} \pm \mathbf{0 . 0 0}$ \\
\hline
\end{tabular}

Table 3: Percent deviation from the lowest mean discounted cost (DC*) for Problem Setting 1 (Base Case) assuming different discrete service time distributions. The bold font indicates the policy that provides the best value for each probability distribution.

\begin{tabular}{ccccc}
\hline & \multicolumn{4}{c}{ Probability Distributions } \\
& Geometric & Neg. Binomial & Poisson & Uniform \\
\hline AOPD & $0.8 \%$ & $0.7 \%$ & $0.7 \%$ & $1.0 \%$ \\
AOPS & $\mathbf{0 . 0 \%}$ & $\mathbf{0 . 0 \%}$ & $\mathbf{0 . 0 \%}$ & $\mathbf{0 . 0 \%}$ \\
FAS & $60.3 \%$ & $72.5 \%$ & $79.3 \%$ & $76.3 \%$ \\
M & $32.9 \%$ & $38.4 \%$ & $41.2 \%$ & $39.9 \%$ \\
\hline DC $^{*}$ & $42809 \pm 767$ & $33802 \pm 699$ & $29878 \pm 674$ & $30989 \pm 708$ \\
\hline
\end{tabular}

stochastic version is perhaps surprising but also encouraging as it implies that advance schedules based on average service times are in fact reasonably effective. Below we present a number of scenarios to determine if similar success for the deterministic case can be seen in other settings.

\subsection{Problem Setting 2: Increased Difference between Service Classes}

In the second setting, we increase the difference in the expected service time and in the standard deviation of the service time between classes. We now consider a system with regular-hour capacity of 14 appointment slots per day. The overtime capacity is 7 appointment slots per day. The system divides demand into only two service classes, with expected service times of 2 and 5 appointment slots and standard deviations of the service time of 1.01 and 3.03 appointment slots, respectively. Thus, one class has a relatively short service time without much variance while the other has a significantly higher average service time and variance. Both service classes are assumed to have negative binomial service time distributions. Demand from each priority class-service class combi- 
nation is assumed to be Poisson with means given in Table 4. All the other parameters remain the same as in the Base Case scenario.

Table 4: Arrival rate for each priority class-service class combination for Problem Setting 2.

\begin{tabular}{cccc}
\hline \multirow{2}{*}{ Priority Class } & \multicolumn{3}{c}{ Service Class } \\
& 1 & 2 & Total \\
\hline 1 & 1.0 & 1.0 & 2.0 \\
2 & - & 1.0 & 1.0 \\
3 & 1.0 & - & 1.0 \\
\hline Total & 2.0 & 2.0 & 4.0 \\
\hline
\end{tabular}

The intent of choosing this problem setting was to pick a scenario that should more likely lead to differences between the performance of the policies coming from the two versions of our model and yet even here the two policies appear to do equally well with the stochastic version only slightly outperforming the deterministic one (difference of around 3\%). Both continue to outperform the two comparator policies though by smaller margins perhaps due to the more limited flexibility in a setting with only two service classes. The simulation results are summarized in Table 5 .

Table 5: Summary of the simulation results for Problem Setting 2. The bold font indicates the policy (policies) that provides (provide) the best mean performance for each metric in a statistical sense $(\alpha=0.05)$.

\begin{tabular}{cccccc}
\hline \multirow{2}{*}{ Metric } & $\begin{array}{c}\text { Patient } \\
\text { Class }\end{array}$ & AOPD & AOPS & FAS & M \\
\hline DC & - & $34547 \pm 694$ & $\mathbf{3 4 2 8 3} \pm \mathbf{7 0 9}$ & $39287 \pm 1561$ & $35654 \pm 1084$ \\
\hline AC & - & $331.73 \pm 1.82$ & $\mathbf{3 2 7 . 8 8} \pm \mathbf{1 . 8 0}$ & $387.00 \pm 5.50$ & $342.07 \pm 3.27$ \\
\hline ACU & - & $\mathbf{1 4 . 0 0} \pm \mathbf{0 . 0 4}$ & $\mathbf{1 4 . 0 0} \pm \mathbf{0 . 0 4}$ & $13.98 \pm 0.04$ & $13.99 \pm 0.04$ \\
\hline \multirow{2}{*}{ ATFAS } & 1 & $\mathbf{1 . 3 4} \pm \mathbf{0 . 0 1}$ & $1.44 \pm 0.01$ & $1.98 \pm 0.02$ & $1.90 \pm 0.02$ \\
& 2 & $\mathbf{1 . 5 6} \pm \mathbf{0 . 0 1}$ & $1.71 \pm 0.02$ & $3.38 \pm 0.06$ & $3.06 \pm 0.04$ \\
\hline \multirow{3}{*}{ AWT } & 1 & $\mathbf{2 . 2 2} \pm \mathbf{0 . 0 2}$ & $2.45 \pm 0.02$ & $5.77 \pm 0.12$ & $4.44 \pm 0.07$ \\
& 2 & $\mathbf{5 . 7 7} \pm \mathbf{0 . 0 3}$ & $6.26 \pm 0.03$ & $7.54 \pm 0.12$ & $6.66 \pm 0.09$ \\
& 3 & $8.80 \pm 0.05$ & $8.89 \pm 0.05$ & $4.49 \pm 0.12$ & $\mathbf{3 . 8 2} \pm \mathbf{0 . 0 8}$ \\
\hline \multirow{2}{*}{$\mathrm{SL}$} & 1 & $\mathbf{9 9 . 7 2} \pm \mathbf{0 . 0 6}$ & $99.35 \pm 0.08$ & $41.16 \pm 1.54$ & $53.45 \pm 1.22$ \\
& 2 & $99.94 \pm 0.01$ & $\mathbf{9 9 . 9 9} \pm \mathbf{0 . 0 1}$ & $55.59 \pm 1.75$ & $70.97 \pm 1.29$ \\
& 3 & $\mathbf{1 0 0 . 0 0} \pm \mathbf{0 . 0 0}$ & $\mathbf{1 0 0 . 0 0} \pm \mathbf{0 . 0 0}$ & $\mathbf{1 0 0 . 0 0} \pm \mathbf{0 . 0 0}$ & $\mathbf{1 0 0 . 0 0} \pm \mathbf{0 . 0 0}$ \\
\hline
\end{tabular}

\subsection{Problem Setting 3: Varying the Ratio between Idle Time and Overtime Costs}

In the third setting, we set the average service times to be the same between service classes but keep a significant difference in their standard deviations. We consider two service classes, with expected service times of 4 appointment slots each and standard deviations of 0.71 and 2.72 appointment slots, respectively. All the other parameters, including the patient arrival rates, remain the same as in the previous setting. 
The simulation results are summarized in Table 6 . It is worth noting that even though the differences between the comparator and the ADP policies are reduced in these last two settings, the ADP policies continue to provide much higher service levels for the high priority patients. Should the value placed on meeting wait time targets be increased (currently set quite low in comparison to the idle time and overtime costs), the difference in performance between the AOPD and AOPS policies and the comparators would undoubtedly increase accordingly.

Table 7 shows the mean discounted cost for different idle time and overtime cost values for the problem setting described above. Here we see the greatest divergence in performance between the two ADP policies with the greatest discrepancy observed when the idle time and overtime costs are equal. Nonetheless, even in this setting, the differences are less than $4 \%$.

Table 6: Summary of the simulation results for Problem Setting 3. The bold font indicates the policy (policies) that provides (provide) the best mean performance for each metric in a statistical sense $(\alpha=0.05)$

\begin{tabular}{cccccc}
\hline \multirow{2}{*}{ Metric } & $\begin{array}{c}\text { Patient } \\
\text { Class }\end{array}$ & AOPD & AOPS & FAS & M \\
\hline DC & - & $32256 \pm 825$ & $\mathbf{3 1 4 0 7} \pm \mathbf{8 2 8}$ & $36751 \pm 2139$ & $32715 \pm 1394$ \\
\hline AC & - & $302.82 \pm 1.74$ & $\mathbf{2 9 5 . 0 7} \pm \mathbf{1 . 7 3}$ & $361.25 \pm 8.42$ & $310.21 \pm 4.46$ \\
\hline ACU & - & $\mathbf{1 6 . 0 1} \pm \mathbf{0 . 0 4}$ & $\mathbf{1 6 . 0 1} \pm \mathbf{0 . 0 4}$ & $\mathbf{1 5 . 9 9} \pm \mathbf{0 . 0 4}$ & $\mathbf{1 6 . 0 0} \pm \mathbf{0 . 0 4}$ \\
\hline ATFAS & $1 \& 2$ & $\mathbf{1 . 5 0} \pm \mathbf{0 . 0 1}$ & $\mathbf{1 . 5 0} \pm \mathbf{0 . 0 1}$ & $3.67 \pm 0.08$ & $3.34 \pm 0.07$ \\
\hline & 1 & $\mathbf{2 . 2 9} \pm \mathbf{0 . 0 2}$ & $2.51 \pm 0.02$ & $6.37 \pm 0.21$ & $4.92 \pm 0.12$ \\
AWT & 2 & $5.83 \pm 0.03$ & $5.68 \pm 0.04$ & $6.60 \pm 0.20$ & $\mathbf{5 . 5 3} \pm \mathbf{0 . 1 3}$ \\
& 3 & $9.95 \pm 0.05$ & $8.81 \pm 0.06$ & $6.67 \pm 0.19$ & $\mathbf{5 . 7 7} \pm \mathbf{0 . 1 4}$ \\
\hline & 1 & $\mathbf{9 9 . 8 3} \pm \mathbf{0 . 0 6}$ & $99.76 \pm 0.06$ & $33.92 \pm 2.71$ & $45.24 \pm 2.20$ \\
SL & 2 & $\mathbf{9 9 . 9 9} \pm \mathbf{0 . 0 1}$ & $\mathbf{9 9 . 9 9} \pm \mathbf{0 . 0 2}$ & $66.18 \pm 2.45$ & $82.75 \pm 1.37$ \\
& 3 & $\mathbf{1 0 0 . 0 0} \pm \mathbf{0 . 0 0}$ & $\mathbf{1 0 0 . 0 0} \pm \mathbf{0 . 0 0}$ & $\mathbf{1 0 0 . 0 0} \pm \mathbf{0 . 0 0}$ & $\mathbf{1 0 0 . 0 0} \pm \mathbf{0 . 0 0}$ \\
\hline
\end{tabular}

Table 7: Percent difference between the mean discounted cost (DC) associated with the AOPD policy and that associated with the AOPS policy for Problem Setting 3 assuming different idle time and overtime cost values.

\begin{tabular}{ccccc}
\hline \multicolumn{5}{c}{ Overtime Cost $/$ Idle Time Cost } \\
$\mathbf{\$ 1 0 0} / \mathbf{\$ 0}$ & $\mathbf{\$ 1 0 0} / \mathbf{\$ 2 5}$ & $\mathbf{\$ 1 0 0} / \mathbf{\$ 5 0}$ & $\mathbf{\$ 1 0 0} / \mathbf{\$ 7 5}$ & $\mathbf{\$ 1 0 0} / \mathbf{\$ 1 0 0}$ \\
\hline $1.03 \%$ & $1.88 \%$ & $2.70 \%$ & $2.57 \%$ & $2.94 \%$ \\
\hline
\end{tabular}

\subsection{Problem Setting 4: A Practical Application}

Finally, we consider a practical example based on data provided by a medium-size clinic in Ontario, Canada. The clinic divides demand into four priority classes with wait time targets of 4 , 8, 12 and 24 days and three service classes with expected service times of 4, 6 and 8 appointments slots. Each appointment slot is 5 minutes in length. Demand from each priority class-service class 

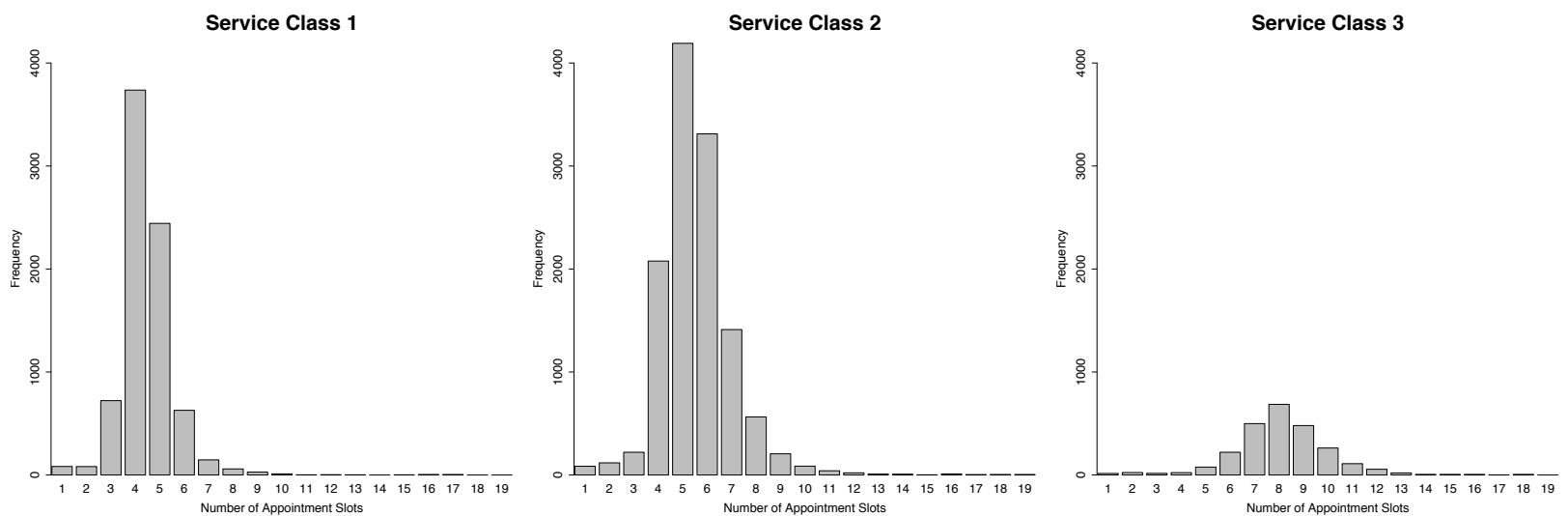

Figure 1: Service time histograms for a practical setting based on data provided by a medium-size clinic in Ontario, Canada. The clinic divides demand into three service classes with mean services times of 20, 30 and 40 minutes, respectively. Each appointment slot is 5 minutes in length.

combination is assumed to be Poisson with arrival rates given in Table 8. Service times follow the empirical discrete probability distributions shown in Figure 1. The regular-hour capacity is set at 144 appointment slots, which is equal to the average daily demand and equivalent to two identical medical resources operating six hours a day. The overtime capacity is 24 appointment slots or one extra hour per medical resource. The postponements penalties are 25, 20,15 and 10 per patient. All the other parameters remain the same as in the previous settings. We assume that no patient is scheduled more than 24 days in advance.

Table 8: Arrival rate for each priority class-service class combination for a practical application.

\begin{tabular}{|c|c|c|c|c|}
\hline \multirow{2}{*}{ Priority Class } & \multicolumn{3}{|c|}{ Service Class } & \multirow{2}{*}{ Total } \\
\hline & 1 & 2 & 3 & \\
\hline 1 & 0.0 & 1.0 & 1.0 & 2.0 \\
\hline 2 & 1.0 & 1.0 & 1.0 & 3.0 \\
\hline 3 & 1.0 & 2.0 & 1.0 & 4.0 \\
\hline 4 & 6.0 & 8.0 & 2.0 & 16.0 \\
\hline Total & 8.0 & 12.0 & 5.0 & 25.0 \\
\hline
\end{tabular}

The corresponding simulation results are summarized in Table 9. For this setting, each policy was simulated for 1000 days with statistics collected for each of 30 simulation runs after a warm-up period of 250 days. The AOPS again shows a slight improvement in the mean discounted cost over the AOPD (around 3.5\%) and both continue to outperform the comparator policies in terms of this metric as well as others. 
Table 9: Summary of the simulation results for Problem Setting 4. The bold font indicates the policy (policies) that provides (provide) the best mean performance for each metric in a statistical sense $(\alpha=0.05)$.

\begin{tabular}{cccccc}
\hline \multirow{2}{*}{ Metric } & $\begin{array}{c}\text { Patient } \\
\text { Class }\end{array}$ & AOPD & AOPS & FAS & M \\
\hline DC & - & $65424 \pm 2944$ & $\mathbf{6 3 2 1 0} \pm \mathbf{2 9 8 2}$ & $140154 \pm 15821$ & $105573 \pm 8156$ \\
\hline AC & - & $536.56 \pm 9.26$ & $\mathbf{5 1 2 . 7 4} \pm \mathbf{9 . 1 7}$ & $1618.69 \pm 183.20$ & $700.96 \pm 29.14$ \\
\hline ACU & - & $142.84 \pm 0.32$ & $142.76 \pm 0.32$ & $142.04 \pm 0.15$ & $\mathbf{1 4 4 . 0 1} \pm \mathbf{0 . 3 4}$ \\
\hline & 1 & $\mathbf{1 . 1 3} \pm \mathbf{0 . 0 2}$ & $1.14 \pm 0.02$ & $2.26 \pm 0.04$ & $2.24 \pm 0.07$ \\
ATFAS & 2 & $\mathbf{1 . 1 5} \pm \mathbf{0 . 0 2}$ & $1.16 \pm 0.03$ & $2.88 \pm 0.06$ & $2.73 \pm 0.10$ \\
& 3 & $\mathbf{1 . 1 6} \pm \mathbf{0 . 0 3}$ & $1.17 \pm 0.03$ & $3.89 \pm 0.12$ & $3.37 \pm 0.13$ \\
\hline & 1 & $\mathbf{1 . 3 0} \pm \mathbf{0 . 0 4}$ & $1.39 \pm 0.05$ & $15.11 \pm 1.26$ & $5.76 \pm 0.30$ \\
$\mathrm{AWT}$ & 2 & $\mathbf{2 . 3 1} \pm \mathbf{0 . 1 5}$ & $2.45 \pm 0.16$ & $15.17 \pm 1.25$ & $6.93 \pm 0.39$ \\
& 3 & $\mathbf{4 . 9 5} \pm \mathbf{0 . 3 1}$ & $5.05 \pm 0.31$ & $15.36 \pm 1.25$ & $7.18 \pm 0.39$ \\
& 4 & $18.93 \pm 0.48$ & $18.74 \pm 0.52$ & $15.70 \pm 1.25$ & $\mathbf{7 . 5 5} \pm \mathbf{0 . 3 9}$ \\
\hline & 1 & $\mathbf{9 9 . 4 1} \pm \mathbf{0 . 1 7}$ & $99.36 \pm 0.18$ & $1.35 \pm 1.93$ & $24.79 \pm 6.22$ \\
$\mathrm{SL}$ & 2 & $\mathbf{9 9 . 8 1} \pm \mathbf{0 . 1 1}$ & $\mathbf{9 9 . 8 2} \pm \mathbf{0 . 1 1}$ & $4.85 \pm 5.98$ & $84.41 \pm 2.34$ \\
& 3 & $\mathbf{9 9 . 9 6} \pm \mathbf{0 . 0 4}$ & $\mathbf{9 9 . 9 8} \pm \mathbf{0 . 0 3}$ & $21.43 \pm 10.91$ & $98.33 \pm 0.67$ \\
& 4 & $\mathbf{1 0 0 . 0 0} \pm \mathbf{0 . 0 0}$ & $\mathbf{1 0 0 . 0 0} \pm \mathbf{0 . 0 0}$ & $\mathbf{1 0 0 . 0 0} \pm \mathbf{0 . 0 0}$ & $\mathbf{1 0 0 . 0 0} \pm \mathbf{0 . 0 0}$ \\
\hline
\end{tabular}

\section{Discussion and Conclusion}

Through this research we have begun to bridge the advance and the appointment scheduling problems - two problems that have been treated separately in past research despite their significant interdependencies.

This paper provides three contributions to the literature. First, we describe a model that schedules patients with varying capacity requirements and urgency levels to a single resource and that can be adapted for either deterministic or stochastic service times. Second, we provide solutions and insights for multiple problem settings assuming deterministic and stochastic appointment durations with extensive numerical analyses. For the deterministic case, we present analytical results that prove the form of the optimal affine approximation in our approach under reasonable conditions. The conditions provide limits on the available capacity in order for the resulting policies to work effectively. For the stochastic version, we provide numerical results that suggest that the deterministic version of the model works reasonably well (within $4 \%$ in our numerical experiments) even in cases where the service time distributions demonstrate significant variability and when service classes vary significantly in both the mean service time and the standard deviation of the service time. Third, we provide an extensive literature review of advance and appointment scheduling.

It is, however, possible that the success of the policies obtained from the deterministic model in (almost) matching the performance of those obtained using the stochastic version may be at- 
tributable to either the use of an affine approximation architecture (possibly curtailing the advantage of incorporating stochastic service times) or the fact that we concentrated our efforts on a setting where within-day waiting/idle time was of little consequence. Thus, two further avenues of research are the implementation of a non-linear approximation architecture similar to Sauré et al. (2015) as well as the incorporation of the sequencing of patients into the MDP model in order to address scenarios where the within-day waiting/idle time is relevant.

\section{Acknowledgements}

This work was partially supported by the Natural Sciences and Engineering Research Council of Canada (NSERC) [grant number 2015-03911].

\section{References}

Astaraky, D., Patrick, J., 2015. A simulation based approximate dynamic programming appraoch to multi-class, multi-resource surgical scheduling. Eur. J. Oper. Res. 245, 309-319.

Bailey, N., 1952. A study of queues and appointment systems in hospital out-patient departments with special reference to waiting times. J. Ro. Stat. Soc. B 14, 185-199.

Begen, M., Levi, R., Queyranne, M., 2012. Technical note - a sampling-based approach to appointment scheduling. Oper. Res. 60, 675-681.

Begen, M., Queyranne, M., 2011. Appointment scheduling with discrete random durations. Math. Oper. Res. 36, 240-257.

Bosch, P., Vanden, P., Dietz, D., Simeoni, J., 1999. Scheduling customer arrivals to a stochastic service system. Nav. Res. Log. 46, 217-229.

Cayirli, T., Kum, K., Quek, S., 2012. A universal appointment rule in the presence of no-shows and walk-ins. Prod. Oper. Manag. 21, 682-697.

Chakraborty, S., Muthuraman, K., Lawley, M., 2010. Sequential clinical scheduling with patient no-shows and general service time distributions. IIE Trans. 42, 354-366.

Chen, R., Robinson, L., 2014. Sequencing and scheduling appointments with potential call-in patients. Prod. Oper. Manag. 23, 1522-1538. 
Denton, B., Gupta, D., 2003. A sequential bounding approach for optimal appointment scheduling. IIE Trans. 35, 1003-1016.

Denton, B., Viapiano, J., Vogl, A., 2007. Optimization of surgery sequencing and scheduling decisions under uncertainty. Health Care Manag. Sc. 10, 13-24.

Dobson, G., Hasija, S., Pinker, E., 2011. Reserving capacity for urgent patients in primary care. Prod. Oper. Manag. 20, 456-473.

Drew, J., Evans, D., Glen, A., Leemis, L., 2008. Computational Probability: Algorithms and Applications in the Mathematical Sciences. Springer Science \& Business Media.

Erdelyi, A., Topaloglu, H., 2009. Computing protection level policies for dynamic capacity allocation problems by using stochastic approximation methods. IIE Trans. 41, 498-510.

Erdogan, S., Denton, B., 2013. Dynamic appointment scheduling of a stochastic server with uncertain demand. INFORMS J. Comput. 25, 116-132.

Feldman, J., Liu, N., Topaloglu, H., Ziya, S., 2014. Appointment scheduling under patient preference and no-show behaviour. Oper. Res. 62, 794-811.

Ge, D., Wan, Z., Zhang, J., 2013. A note on appointment scheduling with piecewise linear costs functions. Math. Oper. Res. 39, 1244-1251.

Gocgun, Y., Ghate, A., 2012. Lagrangian relaxation and constraint generation for allocation and advanced scheduling. Comput. Oper. Res. 39, 2323-2336.

Gocgun, Y., Puterman, M., 2014. Dynamic scheduling with due dates and time windows: an application to chemotherapy patient appointment booking. Health Care Manag. Sc. 17, 60-76.

Green, L., Savin, S., 2008. Reducing delays for medical appointments: A queuning approach. Oper. Res. 56, 1526-1538.

Green, L., Savin, S., Wang, B., 2006. Managing patient service in a diagnostic medical facility. Oper. Res. 54, 11-25.

Guda, H., Dawande, M., Janakiraman, G., Jung, K., 2016. Optimal policy for a stochastic scheduling problem with applications to surgical scheduling. Prod. Oper. Manag. 25, 1194-1202.

Gupta, D., 2007. Surgical suites operations management. Prod. Oper. Manag. 16, 689-700. 
Gupta, D., Denton, B., 2008. Appointment scheduling in health care: challenges and opportunities. IIE Trans. 40, 800-819.

Huang, Y., Zuniga, P., 2012. Dynamic overbooking scheduling system to improve patient access. J. Oper. Res. Soc. 63, 810-820.

Kaandoorp, G., Koole, G., 2007. Optimal outpatient appointment scheduling. Health Care Manag. Sc. 10, 217-229.

Klassen, K., Rholeder, T., 1996. Scheduling outpatient appointments in a dynamic environment. J. Oper. Manag. 14, 83-101.

Klassen, K., Yoogalingam, R., 2008. An assessment of the interruption level of doctors in outpatient appointment scheduling. Oper. Manage. Res. 1, 95-102.

Klassen, K., Yoogalingam, R., 2009. Improving performance in outpatient appointment services with a simulation optimization approach. Prod. Oper. Manag. 18, 447-458.

Klassen, K., Yoogalingam, R., 2014. Strategies for appointment policy design with patient unpunctuality. Decision Sci. 45, 881-911.

Kong, Q., Lee, C., Teo, C., Zheng, Z., 2013. Scheduling arrivals to a stochastic service delivery system using copositive cones. Oper. Res. 61, 711-726.

LaGanga, L., Lawrence, S., 2012. Appointment overbooking in healht care clinics to improve patient service and clinic performance. Prod. Oper. Manag. 21, 874-888.

Liu, N., 2016. Optimal choice for appointment scheduling window under patient no-show behaviour. M\&SOM-Manuf. Serv. Op. 25, 128-142.

Liu, N., Ziya, S., Kulkarni, V., 2009. Dynamic scheduling of outpatient appointments under patient no-shows and cancellations. M\&SOM-Manuf. Serv. Op. 12, 347-364.

Luo, J., Kulkarni, V., Ziya, S., 2012. Appointment scheduling under patient no-shows and service interruptions. M\&SOM-Manuf. Serv. Op. 14, 670-684.

Ma, X., Sauré, A., Puterman, M., Taylor, M., Tyldesley, S., 2016. Capacity planning and appointment scheduling for new patient oncology consults. Health Care Manag. Sc. 19, 347-361. 
Mak, H., Rong, Y., Zhang, J., 2014. Appointment scheduling with limited distributional information. Manage. Sci. 61, 316-334.

Mancilla, C., Storer, R., 2009. Stochastic sequencing and scheduling of an operating room. PhD Thesis, Lehigh University, Department of Industrial and Systems Engineering.

Mancilla, C., Storer, R., 2012. A sample average approximation approach to stochastic appointment sequencing and scheduling. IIE Trans. 35, 655-670.

Mittal, S., Schulz, A.S., Stiller, S., 2014. Robust appointment scheduling, in: Jansen, K., Rolim, J.D.P., Devanur, N.R., Moore, C. (Eds.), Approximation, Randomization, and Combinatorial Optimization. Algorithms and Techniques, pp. 356-370.

Muthuraman, K., Lawley, M., 2008. A stochastic overbooking model for outpatient clnical scheduling with no-shows. IIE Trans. 40, 820-837.

Patrick, J., 2012. A markov decision model for optimal outpatient scheduing. Health Care Manag. Sc. $15,91-102$.

Patrick, J., Puterman, M., Queyranne, M., 2008. Dynamic multi-priority patient scheduling for a diagnostic resource. Oper. Res. 56, 1507-1525.

Robinson, L., Chen, R., 2009. Scheduling doctors' appointments: optimal and empirically based heuristic policies. IIE Trans. 35, 295-307.

Robinson, L., Chen, R., 2010. Traditional and open-access appointment scheduling policies: The effects of patient no-shows. M\&SOM-Manuf. Serv. Op. 12, 330-346.

Samorani, M., LaGanga, L., 2015. Outpatient appointment scheduling given individual daydependent no-show prediction. Eur. J. Oper. Res. 240, 245-257.

Sang, P., Begen, M., Cao, J., 2017. Appointment scheduling with a quantile objective. Unpublished.

Santibáñez, P., Begen, M., Atkins, D., 2007. Surgical block scheduling in a system of hospitals: An application to resource and wait list management in a British Columbia health authority. Health Care Manag. Sc. 10, 269-282.

Santibáñez, P., Chow, V., French, J., Puterman, M., Tyldesley, S., 2009. Reducing patient wait times and improving resource utilization at British Columbia Cancer Agency's ambulatory care unit through simulation. Health Care Manag. Sc. 12, 392-407. 
Sauré, A., Patrick, J., Puterman, M., 2015. Simulation-based approximate policy iteration with generalized logistic functions. INFORMS J. Comput. 27, 579-595.

Sauré, A., Patrick, J., Tyldesley, S., Puterman, M., 2012. Dynamic multi-appointment patient scheduling for radiation therapy. Eur. J. Oper. Res. 223, 573-584.

Schütz, H., Kolisch, R., 2012. Approximate dynamic programming for capacity allocation in the service industry. Eur. J. Oper. Res. 218, 239-250.

Schütz, H., Kolisch, R., 2013. Capacity allocation for demand of different customer-productcombinations with cancellations, no-shows, and overbooking when there is sequential delivery of service. Ann. Oper. Res. 206, 401-423.

Tang, J., Yan, C., Cao, P., 2014. Appointment scheduling algorithm considering routine and urgent patients. Expert Sys. Appl. 41, 4529-4541.

Truong, V., 2015. Optimal advance scheduling. Manage. Sci. 61, 1584-1597.

Tsai, P., Teng, G., 2014. A stochastic appointment scheduling system on multiple resources with dynamice call-in sequence and patient no-shows for an outpatient clinic. Eur. J. Oper. Res. 239, $427-436$.

Wang, P., 1993. Static and dynamic scheduling of customer arrivals to a single-server system. Nav. Res. Log. 40, 345-360.

Wang, W., Gupta, D., 2011. Adaptive appointment systems with patient preferences. M\&SOMManuf. Serv. Op. 13, 373-389.

Weiss, E., 1990. Models for determining estimated start times and case orderings in hospital operating rooms. IIE Trans. 22, 143-150.

White, D., Froehle, C., Klassen, K., 2011. The effect of integrated scheduling and capacity policies on clinical efficiency. Prod. Oper. Manag. 20, 442-455.

Zacharias, C., Pindeo, M., 2014. Appointment scheduing with no-shows and overbooking. Prod. Oper. Manag. 23, 788-801.

Zeng, B., Turkcan, A., Lin, J., Lawley, M., 2009. Clinic scheduling models with overbooking for patients with heterogeneous no-show probabilities. Ann. Oper. Res. 178, 121-144. 


\section{Online Supplement}

Appendix A. Algorithm used to compute the probability distribution of $D(\mathrm{~s}, \mathrm{a})$.

Figure A.1: Pseudo-code of the algorithm used to compute the probability distribution of $D(\mathbf{s}, \mathbf{a})=D_{1 K(\mathbf{s}, \mathbf{a})}$.

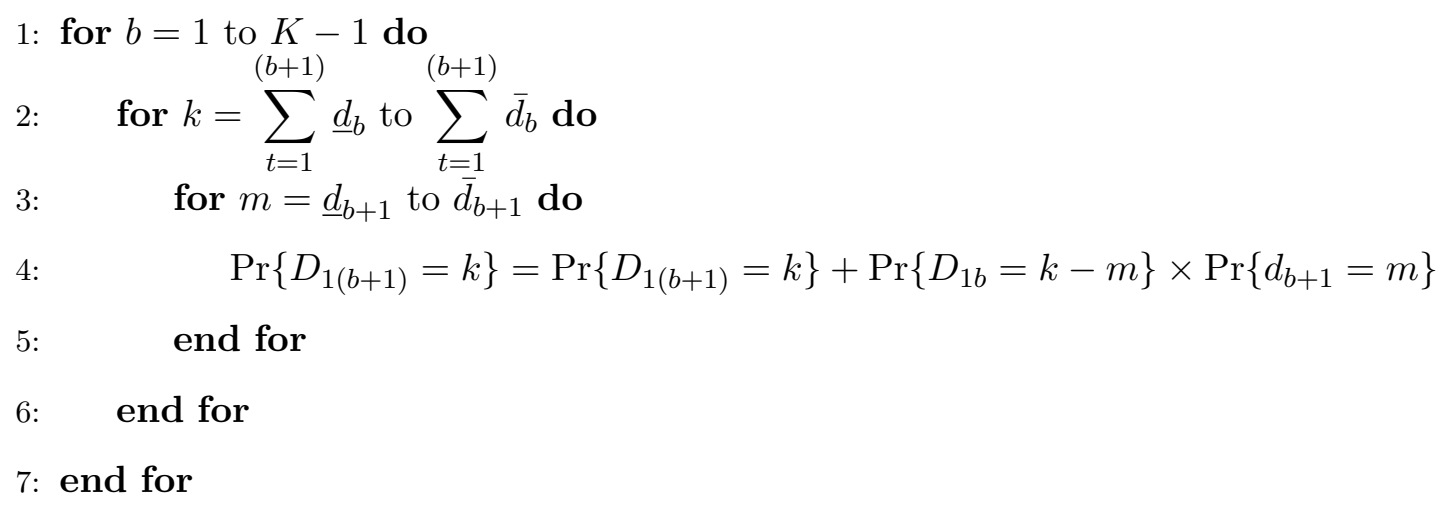




\section{Appendix B. Simulation results for initial states generated using the M policy.}

Table B.1: Summary of the simulation results for Problem Setting 1 (Base Case). The bold font indicates the policy (policies) that provides (provide) the best mean performance for each metric in a statistical sense $(\alpha=0.05)$.

\begin{tabular}{cccccc}
\hline \multirow{2}{*}{ Metric } & $\begin{array}{c}\text { Patient } \\
\text { Class }\end{array}$ & AOPD & AOPS & FAS & M \\
\hline DC & - & $32352 \pm 536$ & $\mathbf{3 2 0 5 5} \pm \mathbf{5 5 9}$ & $50616 \pm 2506$ & $43213 \pm 1525$ \\
\hline AC & - & $313.17 \pm 1.77$ & $\mathbf{3 1 0 . 8 3} \pm \mathbf{1 . 7 1}$ & $578.74 \pm 14.14$ & $435.71 \pm 7.92$ \\
\hline ACU & - & $18.03 \pm 0.05$ & $18.03 \pm 0.05$ & $17.98 \pm 0.05$ & $\mathbf{1 8 . 0 1} \pm \mathbf{0 . 0 5}$ \\
\hline \multirow{3}{*}{ ATFAS } & 1 & $\mathbf{1 . 2 5} \pm \mathbf{0 . 0 1}$ & $1.27 \pm 0.01$ & $2.26 \pm 0.02$ & $2.19 \pm 0.02$ \\
& 2 & $\mathbf{1 . 2 9} \pm \mathbf{0 . 0 1}$ & $1.32 \pm 0.01$ & $2.80 \pm 0.03$ & $2.65 \pm 0.03$ \\
& 3 & $\mathbf{1 . 3 4} \pm \mathbf{0 . 0 1}$ & $1.37 \pm 0.01$ & $3.57 \pm 0.05$ & $3.27 \pm 0.04$ \\
\hline \multirow{2}{*}{ AWT } & 1 & $\mathbf{2 . 0 1} \pm \mathbf{0 . 0 2}$ & $2.17 \pm 0.02$ & $8.56 \pm 0.20$ & $6.23 \pm 0.11$ \\
& 2 & $\mathbf{5 . 5 0} \pm \mathbf{0 . 0 4}$ & $\mathbf{5 . 4 9} \pm \mathbf{0 . 0 4}$ & $8.71 \pm 0.18$ & $6.96 \pm 0.14$ \\
& 3 & $9.47 \pm 0.05$ & $9.63 \pm 0.05$ & $8.56 \pm 0.15$ & $\mathbf{7 . 3 9} \pm \mathbf{0 . 1 4}$ \\
\hline \multirow{2}{*}{ SL } & 1 & $\mathbf{9 9 . 9 8} \pm \mathbf{0 . 0 1}$ & $99.94 \pm 0.02$ & $10.85 \pm 1.81$ & $23.89 \pm 1.87$ \\
& 2 & $\mathbf{1 0 0 . 0 0} \pm \mathbf{0 . 0 0}$ & $\mathbf{1 0 0 . 0 0} \pm \mathbf{0 . 0 0}$ & $38.60 \pm 2.86$ & $68.41 \pm 1.87$ \\
& 3 & $\mathbf{1 0 0 . 0 0} \pm \mathbf{0 . 0 0}$ & $\mathbf{1 0 0 . 0 0} \pm \mathbf{0 . 0 0}$ & $\mathbf{1 0 0 . 0 0} \pm \mathbf{0 . 0 0}$ & $\mathbf{1 0 0 . 0 0} \pm \mathbf{0 . 0 0}$ \\
\hline
\end{tabular}

Table B.2: Percent deviation from the lowest mean discounted cost (DC*) for Problem Setting 1 (Base Case) assuming different discrete service time distributions. The bold font indicates the policy that provides the best value for each probability distribution.

\begin{tabular}{ccccc}
\hline \multirow{2}{*}{ Policy } & \multicolumn{4}{c}{ Probability Distributions } \\
& Geometric & Neg. Binomial & Poisson & Uniform \\
\hline AOPD & $1.0 \%$ & $0.9 \%$ & $0.9 \%$ & $1.5 \%$ \\
AOPS & $\mathbf{0 . 0 \%}$ & $\mathbf{0 . 0 \%}$ & $\mathbf{0 . 0 \%}$ & $\mathbf{0 . 0 \%}$ \\
FAS & $48.4 \%$ & $57.9 \%$ & $63.3 \%$ & $61.3 \%$ \\
M & $30.0 \%$ & $34.8 \%$ & $37.3 \%$ & $36.5 \%$ \\
\hline DC $^{*}$ & $41190 \pm 704$ & $32055 \pm 559$ & $27994 \pm 510$ & $29088 \pm 531$ \\
\hline
\end{tabular}


Table B.3: Summary of the simulation results for Problem Setting 2. The bold font indicates the policy (policies) that provides (provide) the best mean performance for each metric in a statistical sense $(\alpha=0.05)$.

\begin{tabular}{cccccc}
\hline \multirow{2}{*}{ Metric } & $\begin{array}{c}\text { Patient } \\
\text { Class }\end{array}$ & AOPD & AOPS & FAS & M \\
\hline DC & - & $33907 \pm 634$ & $\mathbf{3 3 6 3 6} \pm \mathbf{6 5 1}$ & $37577 \pm 1410$ & $34579 \pm 984$ \\
\hline AC & - & $331.25 \pm 1.81$ & $\mathbf{3 2 7 . 4 0} \pm \mathbf{1 . 7 9}$ & $385.34 \pm 5.44$ & $341.16 \pm 3.24$ \\
\hline ACU & - & $\mathbf{1 3 . 9 9} \pm \mathbf{0 . 0 4}$ & $\mathbf{1 3 . 9 9} \pm \mathbf{0 . 0 4}$ & $13.97 \pm 0.04$ & $13.98 \pm 0.04$ \\
\hline \multirow{2}{*}{ ATFAS } & 1 & $\mathbf{1 . 3 4} \pm \mathbf{0 . 0 1}$ & $1.43 \pm 0.01$ & $1.98 \pm 0.02$ & $1.90 \pm 0.02$ \\
& 2 & $\mathbf{1 . 5 6} \pm \mathbf{0 . 0 1}$ & $1.70 \pm 0.02$ & $3.37 \pm 0.06$ & $3.05 \pm 0.04$ \\
\hline & 1 & $\mathbf{2 . 2 2} \pm \mathbf{0 . 0 2}$ & $2.44 \pm 0.02$ & $5.73 \pm 0.12$ & $4.43 \pm 0.07$ \\
AWT & 2 & $\mathbf{5 . 7 6} \pm \mathbf{0 . 0 3}$ & $6.26 \pm 0.03$ & $7.51 \pm 0.12$ & $6.64 \pm 0.09$ \\
& 3 & $8.79 \pm 0.05$ & $8.88 \pm 0.05$ & $4.47 \pm 0.12$ & $\mathbf{3 . 8 0} \pm \mathbf{0 . 0 8}$ \\
\hline & 1 & $\mathbf{9 9 . 7 6} \pm \mathbf{0 . 0 5}$ & $99.40 \pm 0.08$ & $41.57 \pm 1.55$ & $53.68 \pm 1.22$ \\
SL & 2 & $99.95 \pm 0.01$ & $\mathbf{1 0 0 . 0 0} \pm \mathbf{0 . 0 0}$ & $56.16 \pm 1.77$ & $71.30 \pm 1.28$ \\
& 3 & $\mathbf{1 0 0 . 0 0} \pm \mathbf{0 . 0 0}$ & $\mathbf{1 0 0 . 0 0} \pm \mathbf{0 . 0 0}$ & $\mathbf{1 0 0 . 0 0} \pm \mathbf{0 . 0 0}$ & $\mathbf{1 0 0 . 0 0} \pm \mathbf{0 . 0 0}$ \\
\hline
\end{tabular}

Table B.4: Summary of the simulation results for Problem Setting 3. The bold font indicates the policy (policies) that provides (provide) the best mean performance for each metric in a statistical sense $(\alpha=0.05)$.

\begin{tabular}{cccccc}
\hline \multirow{2}{*}{ Metric } & $\begin{array}{c}\text { Patient } \\
\text { Class }\end{array}$ & AOPD & AOPS & FAS & M \\
\hline DC & - & $31293 \pm 696$ & $\mathbf{3 0 3 6 4} \pm \mathbf{7 0 7}$ & $34006 \pm 1844$ & $31131 \pm 1261$ \\
\hline AC & - & $302.11 \pm 1.71$ & $\mathbf{2 9 4 . 2 9} \pm \mathbf{1 . 7 1}$ & $357.80 \pm 8.21$ & $308.73 \pm 4.39$ \\
\hline ACU & - & $\mathbf{1 5 . 9 9} \pm \mathbf{0 . 0 4}$ & $\mathbf{1 5 . 9 9} \pm \mathbf{0 . 0 4}$ & $15.98 \pm 0.04$ & $\mathbf{1 5 . 9 9} \pm \mathbf{0 . 0 4}$ \\
\hline ATFAS & $1 \& 2$ & $\mathbf{1 . 4 9} \pm \mathbf{0 . 0 1}$ & $\mathbf{1 . 4 9} \pm \mathbf{0 . 0 1}$ & $3.64 \pm 0.08$ & $3.32 \pm 0.06$ \\
\hline & 1 & $\mathbf{2 . 2 9} \pm \mathbf{0 . 0 2}$ & $2.50 \pm 0.02$ & $6.29 \pm 0.21$ & $4.89 \pm 0.12$ \\
AWT & 2 & $5.83 \pm 0.03$ & $5.67 \pm 0.04$ & $6.53 \pm 0.20$ & $\mathbf{5 . 4 9} \pm \mathbf{0 . 1 3}$ \\
& 3 & $9.94 \pm 0.05$ & $8.80 \pm 0.06$ & $6.61 \pm 0.19$ & $\mathbf{5 . 7 3} \pm \mathbf{0 . 1 4}$ \\
\hline & 1 & $\mathbf{9 9 . 8 9} \pm \mathbf{0 . 0 4}$ & $99.82 \pm 0.05$ & $34.83 \pm 2.66$ & $45.76 \pm 2.19$ \\
SL & 2 & $99.99 \pm 0.00$ & $\mathbf{1 0 0 . 0 0} \pm \mathbf{0 . 0 0}$ & $67.10 \pm 2.42$ & $83.21 \pm 1.34$ \\
& 3 & $\mathbf{1 0 0 . 0 0} \pm \mathbf{0 . 0 0}$ & $\mathbf{1 0 0 . 0 0} \pm \mathbf{0 . 0 0}$ & $\mathbf{1 0 0 . 0 0} \pm \mathbf{0 . 0 0}$ & $\mathbf{1 0 0 . 0 0} \pm \mathbf{0 . 0 0}$ \\
\hline
\end{tabular}

Table B.5: Percent difference between the mean discounted cost (DC) associated with the AOPD policy and that associated with the AOPS policy for Problem Setting 3 assuming different idle time and overtime cost values.

\begin{tabular}{ccccc}
\hline \multicolumn{5}{c}{ Overtime Cost $/$ Idle Time Cost } \\
$\mathbf{\$ 1 0 0} / \mathbf{\$ 0}$ & $\mathbf{\$ 1 0 0} / \mathbf{\$ 2 5}$ & $\mathbf{\$ 1 0 0} / \mathbf{\$ 5 0}$ & $\mathbf{\$ 1 0 0} / \mathbf{\$ 7 5}$ & $\mathbf{\$ 1 0 0} / \mathbf{\$ 1 0 0}$ \\
\hline $1.36 \%$ & $2.25 \%$ & $3.06 \%$ & $2.84 \%$ & $3.13 \%$ \\
\hline
\end{tabular}


Table B.6: Summary of the simulation results for Problem Setting 4. The bold font indicates the policy (policies) that provides (provide) the best mean performance for each metric in a statistical sense $(\alpha=0.05)$.

\begin{tabular}{cccccc}
\hline \multirow{2}{*}{ Metric } & $\begin{array}{c}\text { Patient } \\
\text { Class }\end{array}$ & AOPD & AOPS & FAS & M \\
\hline DC & - & $59376 \pm 1495$ & $\mathbf{5 7 2 8 4} \pm \mathbf{1 4 2 6}$ & $62489 \pm 4128$ & $60674 \pm 3465$ \\
\hline AC & - & $534.09 \pm 8.59$ & $\mathbf{5 0 9 . 9 1} \pm \mathbf{9 . 1 9}$ & $787.80 \pm 91.60$ & $604.83 \pm 27.79$ \\
\hline ACU & - & $141.57 \pm 0.30$ & $141.51 \pm 0.30$ & $141.90 \pm 0.10$ & $\mathbf{1 4 2 . 7 2} \pm \mathbf{0 . 3 2}$ \\
\hline \multirow{3}{*}{ ATFAS } & 1 & $\mathbf{1 . 0 5} \pm \mathbf{0 . 0 1}$ & $\mathbf{1 . 0 5} \pm \mathbf{0 . 0 1}$ & $2.15 \pm 0.07$ & $2.12 \pm 0.07$ \\
& 2 & $\mathbf{1 . 0 6} \pm \mathbf{0 . 0 1}$ & $\mathbf{1 . 0 6} \pm \mathbf{0 . 0 1}$ & $2.65 \pm 0.11$ & $2.59 \pm 0.10$ \\
& 3 & $\mathbf{1 . 0 7} \pm \mathbf{0 . 0 1}$ & $1.07 \pm 0.02$ & $3.34 \pm 0.19$ & $3.15 \pm 0.15$ \\
\hline \multirow{3}{*}{ AWT } & 1 & $\mathbf{1 . 1 8} \pm \mathbf{0 . 0 3}$ & $1.23 \pm 0.04$ & $8.40 \pm 1.02$ & $5.76 \pm 0.38$ \\
& 2 & $\mathbf{1 . 9 3} \pm \mathbf{0 . 1 3}$ & $2.02 \pm 0.13$ & $8.46 \pm 1.01$ & $6.29 \pm 0.47$ \\
& 3 & $\mathbf{4 . 0 4} \pm \mathbf{0 . 2 7}$ & $\mathbf{4 . 0 5} \pm \mathbf{0 . 2 7}$ & $8.64 \pm 1.02$ & $6.46 \pm 0.47$ \\
& 4 & $17.09 \pm 0.45$ & $16.65 \pm 0.49$ & $9.01 \pm 1.02$ & $\mathbf{6 . 8 2} \pm \mathbf{0 . 4 7}$ \\
\hline \multirow{2}{*}{$\mathrm{SL}$} & 1 & $\mathbf{1 0 0 . 0 0} \pm \mathbf{0 . 0 0}$ & $\mathbf{9 9 . 9 9} \pm \mathbf{0 . 0 1}$ & $14.24 \pm 8.71$ & $24.61 \pm 8.11$ \\
& 2 & $\mathbf{1 0 0 . 0 0} \pm \mathbf{0 . 0 1}$ & $\mathbf{1 0 0 . 0 0} \pm \mathbf{0 . 0 0}$ & $49.44 \pm 12.79$ & $92.41 \pm 2.34$ \\
& 3 & $\mathbf{1 0 0 . 0 0} \pm \mathbf{0 . 0 0}$ & $\mathbf{1 0 0 . 0 0} \pm \mathbf{0 . 0 0}$ & $88.08 \pm 7.07$ & $\mathbf{1 0 0 . 0 0} \pm \mathbf{0 . 0 0}$ \\
& 4 & $\mathbf{1 0 0 . 0 0} \pm \mathbf{0 . 0 0}$ & $\mathbf{1 0 0 . 0 0} \pm \mathbf{0 . 0 0}$ & $\mathbf{1 0 0 . 0 0} \pm \mathbf{0 . 0 0}$ & $\mathbf{1 0 0 . 0 0} \pm \mathbf{0 . 0 0}$ \\
\hline
\end{tabular}




\section{Appendix C. A proof of the form of the optimal affine value function approximation in the case with deterministic service times}

We present a proof of the form of the optimal affine value function approximation in the case with deterministic service times. The wait time targets, $T(i)$, for each priority class are assumed to increase with $i$ as a high priority patient is, by definition, a patient who must be served sooner. For completeness, we restate the theorem before giving its proof.

\section{Restating the Theorem}

Assuming that $T(i)$ is non-decreasing in $i$, that the wait time penalties are non-decreasing in $n$ and non-increasing in $i$, and that the following conditions are satisfied:

$$
\begin{gathered}
f^{D}(i)>\left(\gamma^{T(i)-1}-\gamma^{T(i)}\right) \mu_{j} h \quad \forall(i, j) \in[I] \times[J] \\
\sum_{j \in J} \mu_{j}\left[\sum_{i=1}^{I} \frac{\gamma^{T(i)-1} I(T(i)>1) \lambda_{i j}}{1-\gamma}+\sum_{m=1}^{N} \gamma^{m-1} E_{\alpha}\left[X_{j m}\right]\right]>\frac{C^{R}}{1-\gamma} \\
\sum_{j \in J} \mu_{j}\left[\sum_{i=1}^{I} \frac{\gamma^{T(i)-n} I(T(i)>n) \lambda_{i j}}{1-\gamma}+\sum_{m=n}^{N} \gamma^{m-n} E_{\alpha}\left[X_{j m}\right]\right]<\frac{C^{R}+C^{O T}}{1-\gamma} \quad \forall n \in[N]
\end{gathered}
$$

Then, the optimal affine value function approximation for the discounted MDP will be given by:

$$
\begin{aligned}
& V_{j n}^{*}= \begin{cases}\mu_{j} h, & \mathrm{n}=1 ; \\
\gamma V_{j(n-1)}^{*}, & 2 \leq n \leq N-1 ; \\
0, & n=N .\end{cases} \\
& W_{i j}^{*}= \begin{cases}V_{j T(i)}^{*}, & \lambda_{i j}>0 ; \\
0, & \lambda_{i j}=0 .\end{cases} \\
& V_{0}^{*}=\frac{1}{1-\gamma}\left(\sum_{\substack{(i, j) \in \\
[I] \times[J]}} \gamma^{T(i)} \mu_{j} h E\left[Y_{i j}\right]+h C^{R}\right),
\end{aligned}
$$

where $\mu_{j}$ is the number of time units required by a patient of service class $j, h$ is the overtime cost per time unit, $\gamma$ is the daily discount factor, $\lambda_{i j}$ is the expected demand from priority class $i$ and service class $j$ patients, and $C^{R}$ is the system regular-hour capacity in time units. 


\section{The Proof}

The outline of the proof is as follows:

1. Prove the primal feasibility of the proposed solution.

2. Determine necessary and sufficient conditions under which a dual solution, together with the proposed primal solution, would satisfy complementary slackness.

3. Demonstrate that there exists a dual solution satisfying the necessary and sufficient conditions.

The existence of a dual solution that together with the proposed primal solution satisfies complementary slackness is sufficient to prove the optimality.

\section{Proving Primal feasibility}

We begin by proving the feasibility of the hypothesized primal solution. Clearly, it gives nonnegative values for $\vec{V}$ and $\vec{W}$. With a little algebraic manipulation, the constraint for the primal LP can be written as:

$$
\begin{aligned}
(1-\gamma) V_{0} \leq & f^{A S}(\mathbf{s}, \mathbf{a})+\sum_{\substack{(i, j, n) \in \\
[I] \times[J] \times[N]}}\left(f^{W T}(i, n)+\gamma V_{j, n-1}-\gamma W_{i j}-f^{D}(i)\right) a_{i j n} \\
& +\sum_{\substack{(j, n) \in \in \\
[J] \times[N]}}\left(\gamma V_{j, n-1}-V_{j n}\right) x_{j n}+\sum_{\substack{(i, j) \in \in \\
[I] \times[J]}}\left(\gamma W_{i j}+f^{D}(i)-W_{i j}\right) y_{i j}+\gamma \sum_{\substack{i, j) \in \\
[I] \times[J]}} W_{i j} E\left[Y_{i j}\right]
\end{aligned}
$$

For state-action pairs for which $\sum_{j \in[J]} \mu_{j}\left(x_{j 1}+\sum_{i \in[I]} a_{i j 1}\right)>C^{R}$, this equates to:

$$
\begin{aligned}
(1-\gamma) V_{0} \leq & +\sum_{\substack{(i, j, n) \in \\
[I] \times[J] \times[N]}}\left(f^{W T}(i, n)+h \mu_{j} I(n=1)+\gamma V_{j, n-1}-\gamma W_{i j}-f^{D}(i)\right) a_{i j n} \\
& +\sum_{\substack{(j, n) \in \\
[J] \times[N]}}\left(h \mu_{j} I(n=1)+\gamma V_{j, n-1}-V_{j n}\right) x_{j n}+\sum_{\substack{(i, j) \in \in \\
[I] \times[J]}}\left(\gamma W_{i j}+f^{D}(i)-W_{i j}\right) y_{i j} \\
& +\gamma \sum_{\substack{(i, j) \in \\
[I] \times[J]}} W_{i j} E\left[Y_{i j}\right]-h C^{R}
\end{aligned}
$$

If we substitute into the above equation the hypothesized solution for the approximate value function, we get: 


$$
\begin{aligned}
(1-\gamma) V_{0} \leq & \sum_{\substack{(i, j, n) \in \\
[I] \times[J] \times[N]}}\left(f^{W T}(i, n)+h \mu_{j} I(n=1)+\gamma^{n-1} \mu_{j} h-\gamma^{T(i)} \mu_{j} h-f^{D}(i)\right) a_{i j n} \\
& +\sum_{\substack{(j, n) \in \in \\
[J] \times[N]}}\left(\gamma^{T(i)} \mu_{j} h+f^{D}(i)-\gamma^{T(i)-1} \mu_{j} h\right) y_{i j}+\gamma \sum_{\substack{(i, j) \in \\
[I] \times[J]}} W_{i j} E\left[Y_{i j}\right]-h C^{R}
\end{aligned}
$$

Since the coefficient of $y_{i j}$ is positive by Equation (C.1), we can substitute $\sum_{n \in[N]} a_{i j n}=y_{i j}$ to get:

$$
\begin{aligned}
(1-\gamma) V_{0} \leq & \sum_{\substack{(i, j, n) \in \\
[I] \times[J] \times[N]}}\left(f^{W T}(i, n)+h \mu_{j} I(n=1)+\left(\gamma^{n-1}-\gamma^{T(i)-1}\right) \mu_{j} h\right) a_{i j n} \\
& +\sum_{\substack{i, j \in \\
[I] \times[J]}} \gamma^{T(i)} \mu_{j} h E\left[Y_{i j}\right]-h C^{R}
\end{aligned}
$$

Again, by Equation (C.1), we can see that the coefficient of $a_{i j n}$ is greater than 0 with equality only if $n=T(i)$. Thus, for state-action pairs that satisfy $\sum_{j \in[J]} \mu_{j}\left(x_{11}+\sum_{i \in[I]} a_{i j 1}\right)>C^{R}$ the state action pair that provides the minimum of the right-hand side of the constraint for the primal LP will have $a_{i j n}=0$ for all $n \neq T(i)$ and yield:

$$
V_{0} \leq \frac{1}{1-\gamma}\left(\sum_{\substack{(i, j) \in \\[J] \times[N]}} \gamma^{T(i)} \mu_{j} h E\left[Y_{i j}\right]-h C^{R}\right)
$$

which is true with equality for the hypothesized value of $V_{0}$.

For state-action pairs where $\sum_{j \in[J]} \mu_{j}\left(x_{j 1}+\sum_{i \in[I]} a_{i j 1}\right) \leq C^{R}$ the primal constraint simplifies to:

$$
\begin{aligned}
(1-\gamma) V_{0} & \leq \sum_{\substack{(i, j, n) \in \\
[I] \times[J] \times[N]}}\left(f^{W T}(i, n)+h \mu_{j} I(n=1)+\left(\gamma^{n-1}-\gamma^{T(i)}\right) \mu_{j} h\right) a_{i j n} \\
& +\sum_{j \in[J]}\left(-(u+h) \mu_{j}\right) x_{j 1}+\sum_{\substack{(i, j) \in \\
[I] \times[J]}} \gamma^{T(i)} \mu_{j} h E\left[Y_{i j}\right]+u C^{R}
\end{aligned}
$$

The right-hand side takes its minimum value when $\sum_{j \in[J]} \mu_{j} x_{j 1}=C^{R}$ and yields: 


$$
V_{0} \leq \frac{1}{1-\gamma}\left(\sum_{\substack{(i, j) \in \in \\[J] \times[N]}} \gamma^{T(i)} \mu_{j} h E\left[Y_{i j}\right]-h C^{R}\right)
$$

This is the same lower bound for $V_{0}$ as in Equation (C.7) and thus is satisfied by the hypothesized value with equality when $\sum_{j \in[J]} \mu_{j} x_{j 1}=C^{R}$. This proves the primal feasibility of the hypothesized solution under the given conditions with tight constraints for state-action pairs where:

$$
\begin{aligned}
\sum_{j \in[J]} \mu_{j} x_{j 1} & \geq C^{R} \\
\sum_{n \in[N]} a_{i j n} & =y_{i j} \quad \forall(i, j) \in[I] \times[J], \text { and } \\
a_{i j n} & =0 \quad \forall(i, j) \in[I] \times[J] \text { and } n \neq T(i)
\end{aligned}
$$

We can now turn to the second stage of the proof where we demonstrate the existence of a dual solution that together with the hypothesized primal solution satisfies complementary slackness. To do so, we need to demonstrate the existence of a dual solution that is zero for all state-action pairs that do not satisfy the Conditions (C.8) to (C.10) and for which all the dual constraints are tight (since all the primary variables are non-zero). To ease the proof we impose the further condition that a dual variable is positive for a given state-action pair only if $\mu_{j} x_{j n}$ and $\mu_{j} a_{i j n}$ equal either zero or $C^{R}+C^{O T}$ for all $i, j$ and $n>1$. We first re-state the dual constraints:

$$
\begin{array}{r}
(1-\gamma) \sum_{\substack{(\mathbf{s}, \mathbf{a}) \in \\
S \times A(s)}} X(\mathbf{s}, \mathbf{a})=1 \\
\sum_{\substack{(\mathbf{s}, \mathbf{a}) \in \\
S \times A(s)}} X(\mathbf{s}, \mathbf{a})\left(x_{j n}-\gamma x_{j, n+1}-\gamma \sum_{i \in[I]} a_{i j, n+1}\right)=E_{\alpha}\left[X_{j n}\right] \\
\sum_{\substack{(\mathbf{s}, \mathbf{a}) \in \\
S \times A(s)}} X(\mathbf{s}, \mathbf{a})\left(y_{i j}-\gamma E\left[Y_{i j}\right]\right)=E_{\alpha}\left[Y_{i j}\right]
\end{array}
$$

We let $B=\{(\mathbf{s}, \mathbf{a}) \in S \times A(\mathbf{s}) \mid X(\mathbf{s}, \mathbf{a})>0\}$. For $n=N$ and imposing the conditions above on the dual solution, Equation (C.12) yields: 


$$
\begin{array}{r}
\sum_{(\mathbf{s}, \mathbf{a}) \in B} X(\mathbf{s}, \mathbf{a}) x_{j N}=E_{\alpha}\left[X_{j N}\right] \quad \forall j \in[J] \\
\Rightarrow \sum_{\substack{(\mathbf{s}, \mathbf{a}) \in B \\
X_{j N}>0}}=\frac{E_{\alpha}\left[X_{j N}\right]}{C^{R}+C^{O T}}
\end{array}
$$

Proceeding similarly for $n=N-1$, Equation (C.12) yields:

$$
\begin{aligned}
\sum_{(\mathbf{s}, \mathbf{a}) \in B} X(\mathbf{s}, \mathbf{a}) & \left(X_{j, N-1}-\alpha X_{j N}\right)=E_{\alpha}\left[X_{j, N-1}\right] \\
& \Rightarrow \sum_{\substack{(\mathbf{s}, \mathbf{a}) \in B \\
X_{j, N-1}>0}} X(\mathbf{s}, \mathbf{a}) x_{j, N-1}=E_{\alpha}\left[X_{j, N-1}\right]+\alpha \sum_{\substack{(\mathbf{s}, \mathbf{a}) \in B \\
X_{j N}>0}} X(\mathbf{s}, \mathbf{a}) x_{j N} \\
& \Rightarrow \sum_{\substack{(\mathbf{s}, \mathbf{a}) \in B \\
X_{j, N-1}>0}} X(\mathbf{s}, \mathbf{a})=\frac{\mu_{j}}{C^{R}+C^{O T}}\left(E_{\alpha}\left[X_{j, N-1}\right]+\gamma E_{\alpha}\left[X_{j N}\right]\right)
\end{aligned}
$$

Proceeding similarly, for all $n>T(I)-1$ we get:

$$
\sum_{\substack{(\mathbf{s}, \mathbf{a}) \in B \\ X_{j n}>0}} X(\mathbf{s}, \mathbf{a})=\frac{\mu_{j}}{C^{R}+C^{O T}} \sum_{m=n}^{N} \gamma^{m-n} E_{\alpha}\left[X_{j m}\right]
$$

For $n=T(I)-1$ there is the added complication that $a_{I j, T(I)}$ may be non-zero. Equation (C.12) now yields:

$$
\sum_{(\mathbf{s}, \mathbf{a}) \in B} X(\mathbf{s}, \mathbf{a})\left(x_{j, T(I)-1}-\gamma\left(x_{j, T(I)}+a_{I j, T(I)}\right)\right)=E_{\alpha}\left[X_{j, T(I)-1}\right]
$$

which implies that:

$$
\sum_{\substack{(\mathbf{s}, \mathbf{a}) \in B \\ X_{j, T(I)-1}>0}} X(\mathbf{s}, \mathbf{a}) x_{j, T(I)-1}=E_{\alpha}\left[X_{j, T(I)-1}\right]+\gamma \sum_{\substack{(\mathbf{s}, \mathbf{a}) \in B \\ X_{j, T(I)}>0}} X(\mathbf{s}, \mathbf{a}) x_{j, T(I)}+\gamma \sum_{\substack{(\mathbf{s}, \mathbf{a}) \in B \\ a_{I j, T(I)}>0}} X(\mathbf{s}, \mathbf{a}) a_{I j, T(I)}
$$

However, Equations (C.11) and (C.13) yield: 


$$
\sum_{\substack{(\mathbf{s}, \mathbf{a}) \in B \\ a_{I j, T(I)}>0}} X(\mathbf{s}, \mathbf{a}) a_{I j, T(I)}=\sum_{\substack{(\mathbf{s}, \mathbf{a}) \in B \\ a_{I j, T(I)}>0}} X(\mathbf{s}, \mathbf{a}) y_{I j}=E_{\alpha}\left[Y_{I j}\right]+\frac{\lambda_{I j}}{1-\gamma}
$$

where $\lambda_{I j}$ is the arrival rate for patients of priority class $I$ and service class $j$.

Assuming $\mu_{j} y_{I j}=C^{R}+C^{O T}$ and $E_{\alpha}\left[Y_{I j}\right]$ is set equal to $\lambda_{I j}$ we get:

$$
\sum_{\substack{(\mathbf{s}, \mathbf{a}) \in B \\ a_{I j, T(I)}>0}} X(\mathbf{s}, \mathbf{a})=\frac{1}{1-\gamma}\left(\frac{\mu_{j} \lambda_{I j}}{C^{R}+C^{O T}}\right)
$$

Thus, substituting back into Equation (C.14) we get:

$$
\sum_{\substack{(\mathbf{s}, \mathbf{a}) \in B \\ x_{j, T(I)-1}>0}} X(\mathbf{s}, \mathbf{a})=\frac{\mu_{j}}{C^{R}+C^{O T}}\left(\sum_{m=T(I)-1}^{N} \gamma^{m-T(I)+1} E_{\alpha}\left[X_{j m}\right]+\frac{\gamma}{1-\gamma} \lambda_{I j}\right)
$$

Thus, in general, for $n>1$ we get:

$$
\sum_{\substack{(\mathbf{s}, \mathbf{a}) \in B \\ x_{j n}>0}} X(\mathbf{s}, \mathbf{a})=\frac{\mu_{j}}{C^{R}+C^{O T}}\left(\sum_{m=n}^{N} \gamma^{m-n} E_{\alpha}\left[X_{j m}\right]+\sum_{i=1}^{I} \frac{\gamma^{T(i)-n}}{1-\gamma} I(n<T(i)) \lambda_{i j}\right)
$$

For $n=1$, Equation (C.12) still yields:

$$
\begin{aligned}
\sum_{\substack{(\mathbf{s}, \mathbf{a}) \in B \\
x_{j 1}>0}} X(\mathbf{s}, \mathbf{a}) x_{j 1} & =E_{\alpha}\left[X_{j 1}\right]+\gamma \sum_{\substack{(\mathbf{s}, \mathbf{a}) \in B \\
x_{j 2}>0}} X(\mathbf{s}, \mathbf{a}) x_{j 2} \\
& =\sum_{m=1}^{N} \gamma^{m-1} E_{\alpha}\left[X_{j m}\right]+\sum_{i=1}^{I} \frac{\gamma^{T(i)-1}}{1-\gamma} I(1<T(i)) \lambda_{i j}
\end{aligned}
$$

However, unlike for the cases with $n>1$ we cannot simply set $x_{j 1}$ equal to $C^{R}+C^{O T}$ or 0 since we need to enforce by Condition (C.8) that $\sum_{j \in J} \mu_{j} x_{j 1} \geq C^{R}$ for all $(\mathbf{s}, \mathbf{a}) \in B$. Thus, along with satisfying Equation (C.16), our dual solution must also satisfy:

$$
\begin{array}{r}
\sum_{(\mathbf{s}, \mathbf{a}) \in B} X(\mathbf{s}, \mathbf{a}) \sum_{j \in J} \mu_{j} x_{j 1} \geq \frac{C^{R}}{1-\gamma} \\
\left.\Rightarrow \sum_{j \in[J]} \mu_{j}\left(\sum_{m=1}^{N} \gamma^{m-1} E_{\alpha}\left[X_{j m}\right]+\sum_{i=1}^{I} \frac{\gamma^{T(i)-1}}{1-\gamma} I(1<T(i)) \lambda_{i j}\right)\right) \geq \frac{C^{R}}{1-\gamma}
\end{array}
$$

which is enforced by Condition (C.2). 


\section{The Existence of a Dual Solution Satisfying Complementary Slackness}

The above argument suggests a weighting scheme for a dual feasible solution that, together with the proposed primal solution, satisfies complementary slackness. It remains to prove that a dual solution satisfying the above weighting scheme must exist. We can determine the state-action pairs with positive dual weight starting on day 1 and working up to day $N$. A dual solution exists if:

1. The total dual weight available $\frac{1}{1-\gamma}$ given by Equation (C.11) does not exceed the combined weight assigned to all states where $x_{j n}$ or $\sum_{i=1}^{I} a_{i j n}$ are greater than zero for any $n$.

2. The total weight assigned to dual variables where $\sum_{n=1}^{N} a_{i j n}$ is positive and is equal to the weight assigned to dual variables where $y_{i j}$ is positive.

This turns out to be straightforward as it is easy to show that, under the above weighting scheme and using Equation (C.3),

$$
\begin{aligned}
& \sum_{x_{j n}>0}\left(X(\mathbf{s}, \mathbf{a}) \mu_{j} x_{j n}+\sum_{i=1}^{I} \sum_{a_{i j n}>0} X(\mathbf{s}, \mathbf{a}) \mu_{j} a_{i j n}\right) \leq \frac{C_{R}+C^{O T}}{1-\gamma} \\
\Rightarrow & \sum_{x_{n}>0} X(\mathbf{s}, \mathbf{a})+\sum_{i=1}^{I} \sum_{a_{i n}>0} X(\mathbf{s}, \mathbf{a}) \leq \frac{1}{1-\gamma}
\end{aligned}
$$

for all $n \in[N]$ and

$$
\sum_{n=1}^{N} \sum_{\left(\mathbf{s}, \mathbf{a} \in B \mid a_{i j n}>0\right.} X(\mathbf{s}, \mathbf{a}) a_{i j n}=\sum_{\left(\mathbf{s}, \mathbf{a} \in B \mid y_{i j}>0\right.} X(\mathbf{s}, \mathbf{a}) y_{i j}
$$

for all $(i, j) \in[I] \times[J]$. Thus, there exist admissible state-action pairs that satisfy the above weighting scheme proving the existence of the required dual solution. 